\title{
STRUCTURE OF THE COMPLEX OF CYTOCHROME C WITH CARDIOLIPIN IN NON-POLAR ENVIRONMENT
}

\author{
Vladimirov G.K., ${ }^{\mathrm{a}, \mathrm{b}, \mathrm{c} \dagger}$ Vikulina A.S., ${ }^{\mathrm{d}, \mathrm{e}^{* \dagger}}$ Volodkin D.V. ${ }^{\mathrm{d}, \mathrm{e}}$, Vladimirov Yu.A., ${ }^{\mathrm{a}, \mathrm{b}, \mathrm{c}}$
}
${ }^{a}$ M.V. Lomonosov Moscow State University, Faculty of Fundamental Medicine, Leninskiye gory 1-3, 119991 Moscow, Russian Federation

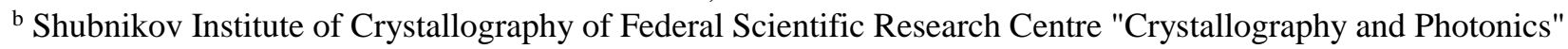 of Russian Academy of Sciences", Leninskiy pr-t 59, 119333 Moscow, Russian Federation
${ }^{c}$ First Moscow State Medical University of the Ministry of Health of the Russian Federation (Sechenov University), Bolshaya Pirogovskaya st. 2-4, 119991, Moscow, Russia Federation
${ }^{\mathrm{d}}$ Nottingham Trent University, School of Science and Technology, Clifton Lane, Nottingham NG11 8NS, UK
${ }^{\mathrm{e}}$ Fraunhofer Institute for Cell Therapy and Immunology, Branch Bioanalytics and Bioprocesses (Fraunhofer IZI- BB), Am Muehlenberg 13, 14476 Potsdam-Golm, Germany
$\dagger$ These authors contributed equally to this work

* Author to whom correspondence should be addressed; e-mail: anna.vikulina@ntu.ac.uk, tel: +44 1158488062

\section{Highlights}

In non-polar medium, complex of cytochrome $c$ with cardiolipin forms nanoparticles of 2 populations - sizes of 7.8 and $12.1 \mathrm{~nm}$

Enzymatic activity of the complex of cytochrome $c$ with cardiolipin in non-polar environment has been shown.

\section{Abstract}

The complex of mitochondrial protein cytochrome $\mathrm{c}(\mathrm{CytC})$ with anionic phospholipid cardiolipin (CL) plays a crucial role in the initiation of apoptosis by catalyzing lipid peroxidation in mitochondrial membranes. In our previous papers, we found that $\mathrm{CytC}$ and CL mixed in millimolar concentrations form a sediment showing microcrystals composed of nanospheres (Cyt-CL) of 11-12 and $8 \mathrm{~nm}$ in diameter. The hypothesis was proposed that Cyt-CL, having hydrophobic shell, may appear inside the membrane lipid bilayer in mitochondria and peroxidase membrane phospholipids so initiating the apoptotic cascade. In this work, Cyt-CL complex dissolved in chloroform or hexane was investigated as a model of the complex in mitochondrial membranes. We used dynamic light scattering method to measure the size of the particles. The analysis of particles size distribution of Cyt-CL in chloroform allows to reveal three dominant diameters of $12.1 \pm 1.4,7.8 \pm 1.0$, and $4.7 \pm$ $0.7 \mathrm{~nm}$. The first two values are closed to those, earlier obtained with small-angle X-ray scattering method in Cyt-CL microcrystals, $11.1 \pm 1.0$ and $8.0 \pm 0.7 \mathrm{~nm}$. CL extracted in chloroform-methanol forms a real solution of particles with diameter of $0.7 \pm 0.1 \mathrm{~nm}$. In methanol-water phase, CL and $\mathrm{CL}+\mathrm{CytC}$ mixture form particles of $83.7 \pm 9.8$ and $71.3 \pm 11.6 \mathrm{~nm}$, respectively. Apparently, cardiolipin in 50\% methanol forms single-layer liposomes regardless of the presence of CytC in the medium. Partial unfolding of $\mathrm{CytC}$ in the complex was evidenced by (a) appearance of fluorescence of tyrosine and tryptophan residues and (b) disappearance of the absorption band at $699 \mathrm{~nm}$ due to breakdown of heme iron - methionine bond $>$ F $\cdots$ S(Met80). In hydrophobic solvent Cyt-CL exhibited quasi-lipoperoxidase and lipoxygenase activity as was shown in kinetic measurements of 
chemiluminescence enhanced by coumarin C-525, a selective sensitizer of chemiluminescence, associated with reactions of lipid peroxyl radicals.

Our data in this model system do not contradict the hypothesis (Vladimirov, Y.A. et al. Biochemistry (Mosc) 78, 1086-1097) that nanospheres of Cyt-CL complex, embedded into the lipid phase of mitochondrial membrane, catalyze lipid peroxidation, thereby initiating apoptosis.

Keywords

Cytochrome $c$; cardiolipin; dynamic light scattering; chemiluminescence

\section{Abbreviations}

Cytochrome $c$ (CytC), cardiolipin (CL), 1,1',2,2'-tetraoleylcardiolipin (TOCL), cardiolipin from bovine heart (BCL), complex of cytochrome $\mathrm{c}$ with cardiolipin (Cyt-CL), small-angle X-ray scattering (SAXS), dynamic light scattering (DLS)

\section{Introduction}

\subsection{The biological function of the complex of $\mathrm{CytC}$ with $\mathrm{CL}$}

Over the past decade, the biological role of the complex of CytC with CL formed in the lipid membranes of mitochondria has attracted significant interest from biologists and chemists. This, for instance, is indicated in the leading research performed in the laboratory of V.E. Kagan at the University of Pittsburgh (Kagan et al., 2009a; Kagan et al., 2004; Kagan et al., 2005; Tyurina et al., 2014). The role of the complex is associated with initiation of apoptosis (Kagan et al., 2009a; Kagan et al., 2004), which is an indispensable process for development, aging and death of living organisms. On the other hand, together with the well-known cytosolic enzymes such as cyclooxygenases and lipoxygenases, the Cyt-CL complex is directly involved into biosynthesis of extremely important metabolic regulators - lipid mediators (Tyurina et al., 2014).

The protein-lipid complex of CytC with CL has recently been the subject of a large number of studies, since the formation of the complex plays a key role in triggering the cascade of reactions in the cell leading to its programmed death, apoptosis (Kagan et al., 2004; Kagan et al., 2005). This main function of Cyt-CL is realized in the lipid bilayer of the mitochondrial membrane. It is accompanied by oxidation of unsaturated fatty acid residues of phospholipids including, in the first place, CL. Eventually, Cyt-CL can reach the site of convergence of the outer and inner mitochondrial membranes, where lipid peroxidation damages the VDAC (Voltage Dependent Anion Channel)ANT (adenine nucleotide translocase) complex. This in turn leads to reduction of the barrier property of the internal membrane, swelling of the matrix, appearance of large pores in the outer membrane, and finally to the release of CytC into cytoplasm that leads to launching of the apoptotic cascade (Skulachev, 1998). While, up to now the structure of the complex and the mechanism of its function are not well understood. Most of researchers support the hypothesis of membrane-bound CytC (Brown and Wuthrich, 1977) and the review (Kagan et al., 2009b). According to this hypothesis, CytC is attached to the surface of the lipid bilayer that results in changing the protein conformation (Brown and Wuthrich, 1977; Kobayashi et al., 2016; Rytomaa and Kinnunen, 1995; Snider et al., 2013; Spooner and Watts, 1991a, b) (Vladimirov et al., 2013b). 


\subsection{Cytochrome $\boldsymbol{c}$ forms with cardiolipin a complex in the form of nanospheres (Cyt-CL)}

In our previous communication, the we showed that the complex of CytC with 1,1',2,2'tetraoleylcardiolipin (TOCL), prepared by mixing a concentrated solution of cytochrome $c$ and TOCL dissolved in small volume of methanol, is poorly soluble in water and precipitates (Vladimirov et al., 2013a; Vladimirov et al., 2011). The SAXS study of the sediment has demonstrated that the complex of CytC with TOCL consists of microcrystals showing interplanar reflection distances of 8.0 and $11.1 \mathrm{~nm}$ (Proskurnina et al., 2013; Shtykova et al., 2013; Vladimirov et al., 2013a; Vladimirov et al., 2011). The spectrophotometric determination of Cyt-CL concentration changes in the supernatant, upon the addition of a given amounts of the cardiolipin, showed that the stoichiometric ratio $\mathrm{CL} / \mathrm{CytC}$ is constant within a wide range of Cyt-CL concentrations and was about 35/1 at $\mathrm{pH}$ 7. These data allowed to assume that the complex Cyt-CL is a nanosphere of approximately $11.2 \mathrm{~nm}$ in diameter, formed by one CytC molecule and a monolayer of CL surrounding this molecule (Vladimirov et al., 2011). At low pH (3.5-5.5) the protein to lipid ratio in Cyt-CL was found to be essentially lower (11-12) than that at neutral medium and that the size of cells in the microcrystals can be equal to $11.1 \pm 1.0 \mathrm{~nm}$ and/or $8.0 \pm 0.7 \mathrm{~nm}$; with the contribution of these two structures depending on pH (Proskurnina et al., 2013; Shtykova et al., 2013; Vladimirov et al., 2013a).

\subsection{Partial unfolding of $\mathrm{CytC}$ in $\mathrm{Cyt}-\mathrm{CL}$ nanoparticles}

Some reports support the hypothesis that the structure of CytC in the Cyt-CL complex differs from that of native CytC (Vladimirov et al., 2013b). The work (Choi and Swanson, 1995) reports an influence of bovine cardiolipin and dioleoylphosphatidylglycerol on the structure of $\mathrm{CytC}$ at the protein:lipid ratio of 1:100. The number of alpha helices decreased by 10 and $5 \%$ and the number of beta-structures increased by 15 and $5 \%$ in the presence of bovine cardiolipin and dioleoylphosphatidylglycerol, respectively. Other authors have shown that the interaction of CytC with CL-containing phospholipid membrane results in partial unfolding of the protein and dissociation of the Met80 from the heme. (Belikova et al., 2006; Kapralov et al., 2007; Mandal et al., 2015; O'Brien et al., 2015; Patriarca et al., 2009; Sinibaldi et al., 2008). A partial unfolding of the CytC macromolecule was supposed to occur also in the nanospheres of Cyt-CL sediment (Vladimirov et al., 2011). Considering the fact that the diameter of the nanosphere is $11.2 \mathrm{~nm}$ and the thickness of two layers of TOCL is approximately $5.6 \mathrm{~nm}$, the size of the CytC globule should be estimated as approximately $5.6 \mathrm{~nm}$, which significantly exceeds the size of the globule of native CytC $(3.5 \mathrm{~nm} \times 3.9 \mathrm{~nm}$, as found in the protein database for the structure of PDB IP 3NBS and our SAXS results (Vladimirov et al., 2011)). This means that the formation of the Cyt-CL complex leads to a remarkable increase in the dimensions of the $\mathrm{CytC}$ molecule inducing its partial melting.

The increase in size of the CytC molecule in the complex with CL is confirmed by analysis of fluorescence of the complex. The addition of CytC to CL leads to appearance of fluorescence of the tyrosine (Tyr) and tryptophan (Trp) residues, which is not observed in native cytochrome (Belikova et al., 2006; Kapralov et al., 2011) due to energy transfer from Tyr or Trp to the heme located nearby. The appearance of fluorescence is caused by an increase in the distance of the residues of Tyr and Trp from the heme due to partial melting of the CytC globule. 


\subsection{Peroxidase activity of Cyt-CL}

In previous studies, we showed that formation of Cyt-CL complex in water leads to the rupture of iron-sulfur bond between heme iron and methionine Met-80 (Belikova et al., 2006; Vladimirov et al., 2011). This bond is absent in other hemoproteins, which have peroxidase activity. The native CytC has this bond that prevents binding of the heme with $\mathrm{H}_{2} \mathrm{O}_{2}$ and therefore the native CytC does not possess the peroxidase activity. It is important to note that this bond has a characteristic absorption peak in the region of $700 \mathrm{~nm}$, which makes it possible to probe the presence of the bond spectrophotometrically. It was shown that when Cyt-CL complex is formed, the structure of an active center of CytC changes, which allows $\mathrm{H}_{2} \mathrm{O}_{2}$ to penetrate the CytC active site (Vladimirov et al., 2006b) and, as a result, facilitates the appearance of peroxidase activity (Belikova et al., 2006). Studying the rupture of iron-methionine bonds in CytC by monitoring its absorption at $700 \mathrm{~nm}$ has revealed a correlation between the extent of the bond breakage and the level of peroxidase activity (Vladimirov et al., 2006c).

The hydrophobic nanoparticles of Cyt-CL perform lipid peroxidation in mitochondrial membranes, most likely directly in the interior of the inner membrane being embedded between the two lipid monolayers forming the lipid bilayer (Vladimirov et al., 2013b). Recently, we have demonstrated that the Cyt-CL complex can be formed in non-polar solvents such as chloroform and hexane (Vikulina et al., 2015) and the complex can catalyze formation of lipid radicals in both quasilipoxygenase and lipoperoxidase reactions (Vladimirov et al., 2017).

\subsection{The purpose of this study}

Self-assembly of Cyt-CL nanoparticles in non-polar environment presents an easy and elegant approach for the mimics of Cyt-CL embedded into the membrane by surrounding of CytC-CL complex by lipophilic phase, e.g. straight-chained saturated hydrocarbons. However, to the best of our knowledge, there is no literature on the structure of the Cyt-CL complex in non-polar environment. The structure of Cyt-CL complex in non-polar environment cannot be deduced from crystal phase and direct evidence of the complexation and analyses would shed light on the complex structure and its vital role in apoptosis. Therefore, the purpose of this study was to investigate the structure of the Cyt-CL complex in non-polar environment through analysis of the size, absorbance, and fluorescence of the complex and to probe its enzymatic activity by chemiluminescent reaction of lipid peroxidation.2. Materials and methods

\subsection{Materials}

1,1',2,2'-tetraoleoyl-cardiolipin and cardiolipin from bovine heart (BCL) were purchased from Avanti Polar Lipids, USA, cytochrome $c$ from horse heart, chloroform ( $\geq 99 \%)$, methanol $(99,8 \%)$ and $n$-hexane $(\geq 99 \%)$ were purchased from Sigma, USA. 2,3,5,6-1H,4H-Tetrahydro-9-(2'benzooxazolyl)-quinolizin-(9,9a,l-gh)coumarin C-525 was purchased from Radiant Dyes Laser Accessories GmbH (Wermelskirchen, Germany). The water used in all experiments was prepared via a Millipore Milli-Q purification system (France) and had a resistivity higher than $18 \mathrm{M} \Omega * \mathrm{~cm}$. 


\subsection{Methods}

\subsubsection{Preparation of Cyt-CL complex in non-polar environment}

Cyt-CL complex was prepared based on the Folch method of lipid extraction (Folch et al., 1957) according to the protocol described in (Vikulina et al., 2015) with slight modifications. First, 260 $\mu \mathrm{M}$ of aqueous-methanol solution (4:1 v/v) of CytC was mixed with $13.5 \mathrm{mM}$ methanol solution of TOCL in order to obtain aqueous-methanol $(1: 1 \mathrm{v} / \mathrm{v})$ complex of CytC with TOCL in a molar ratio of 1:30. At this ratio CL is in excess to $\mathrm{CytC}$ that facilitates the redistribution of Cyt-CL from watermethanol to chloroform phase. (The stoichiometry CL/CytC at $\mathrm{pH}$ 7.0-7.4 is 35-60, depending on pH (Vladimirov et al., 2013a; Vladimirov et al., 2011) and for this reason the enzymatic activity was measured in this work at CL/CytC ratio of 50:1). Then, chloroform was added to the aqueousmethanol Cyt-CL complex to reach a volume ratio of 2:1:1 chloroform:water:methanol. The mixture was intensively stirred by vortexing for 2 minutes. After the spontaneous phase separation, the lower (chloroform-methanol) fraction containing the extracted Cyt-CL complex was carefully taken up with the Pasteur pipette, stored at room temperature and analyzed.

Chloroform-methanol mixture is supposed to extract all lipids (Folch et al., 1957). So, we can assume, that there is no Cyt-CL in the upper phase, but between the two phases there is a precipitate, to which $40 \%$ of Cyt-CL should belong, as in the lower phase we have detected $60 \%$ of the initial CytC amount (measured by absorbency).

\subsubsection{Dynamic light scattering (DLS)}

Measurements were made with a Zetasizer Nano ZS (Malvern, UK) in a quartz cuvette. A number of consecutive measurements are being made in one sample, one after another, with an interval of approximately 3 minutes between successive measurements starts. The result of a typical measurement (Number PSD - the distribution of particle sizes by the proportion (percentage) of particles of the specified size from the total number of particles in the sample) is shown in Fig. 1. (See details in "Results"). Light scattered by the sample was detected at an angle of $173^{\circ}$ with laser source operating at a wavelength of $633 \mathrm{~nm}$. To remove large particles that can interfere with the analysis, the samples were centrifuged during 20 minutes at $14000 \mathrm{~g}$ prior to the measurements. The analysis of the particle size data has been carried out using original Malvern Zetasizer Software. The initial signal received on the device is the intensity of light scattered by particles in the solution, recorded in time. The velocity of the particles in the solution depends on the particle size in accordance with the Stokes law. To estimate the particle size, the instrument sequentially shifts the obtained intensity-time curve by a certain $\Delta \mathrm{t}$, and calculates the correlation coefficient between a curve with a given $\Delta \mathrm{t}$ and the original curve. As a result, we obtain a correlogram on which the correlation coefficient is plotted against $\Delta \mathrm{t}$. From $\Delta \mathrm{t}$, at which the correlation coefficient begins to decrease sharply, the software allows to calculate the distribution of the number of particles in the solution according to their size, based on the intensity of light scattered by the particles. Such a distribution gives a slightly overestimated mean particle size, since larger particles scatter more light (the intensity of scattered light is proportional to the sixth power of the particle diameter). Using Mie's theory, the software can build a Number PSD - the distribution of the number of particles along 
their diameter. In this case, the resulting average particle size determines their dimensions more accurately (Stetefeld et al., 2016).

The following values of the refractive indices at $633 \mathrm{~nm}$ for solvents were used for these calculations: water - 1.3317 (Hale and Querry, 1973), methanol - 1.326 (El-Kashef, 2000), chloroform - 1.442 (Kedenburg et al., 2012).

The refractive indices for water-methanol and chloroform-methanol medium were calculated from the equation:

$$
\frac{n^{2}-1}{n^{2}+2}=\frac{n_{1}{ }^{2}-1}{n_{1}{ }^{2}+2} \varphi_{1}+\frac{n_{2}{ }^{2}-1}{n_{2}{ }^{2}+2} \varphi_{2}
$$

Where $\varphi_{i}$ is the volume fraction of the component, $n$ is the refractive index of the mixture, and $n_{i}$ is the refractive index of the mixture component (Narendra et al., 2011).

The refractive indices of the two-component media used in the present study were: water-methanol $(4: 1 \mathrm{v} / \mathrm{v})-1.3306$, water-methanol $(1: 1 \mathrm{v} / \mathrm{v})-1.3288$, chloroform-methanol $(86: 14 \mathrm{v} / \mathrm{v}$ - according to (Folch et al., 1957)) - 1.4256.

In addition, the following values of the refractive indices of the analyzed particles were used: $\mathrm{CytC}$ - 1.34 (Lee et al., 2002), Cyt-CL complex - 1.46 (refractive index of lipid bilayer (Maniti et al., 2011)). The absorption of at a wavelength of $633 \mathrm{~nm}$ CytC and TOCL can be neglected.

The following values of the dynamic viscosity of solvents were used: water $-0.89 \mathrm{mPa}$ sec (Kestin et al., 1978), water-methanol $(4: 1 \mathrm{v} / \mathrm{v})-1.23 \mathrm{mPa} \mathrm{sec}$, water-methanol $(1: 1 \mathrm{v} / \mathrm{v})-1.61 \mathrm{mPa} \mathrm{sec}$ (Yilmaz, 2002).

For the chloroform-methanol mixture $(86: 14 \mathrm{v} / \mathrm{v})$, the dynamic viscosity value used was $0.696 \mathrm{mPa}$ sec. A change in temperature from $303 \mathrm{~K}$ to $298 \mathrm{~K}$ corresponds to a change in the viscosity of this mixture from 0.6315 (Crabtree and O'Brien 1991) to $0.663 \mathrm{mPa}$ sec (Kadam, et al. 2006). That is, when the temperature drops by 5 degrees, the viscosity increases by $5 \%$. Thus, the extrapolated viscosity at $293 \mathrm{~K}$ will be $0.696 \mathrm{mPa}$ sec.

\subsubsection{Spectrophotometric analysis of conformational changes in CytC}

Spectrophotometric measurements were carried out on a Specord 200 (Analytik Jena, Germany) using quartz cuvettes with an optical path length of $1.00 \mathrm{~cm}$. The spectra were recorded from the total volume of $3 \mathrm{ml}$ in a range of $650-750 \mathrm{~nm}$.

The spectra of $200 \mu \mathrm{M} \mathrm{CytC}$ in aqueous and aqueous-methanol solutions with varied volume ratio were measured. The characteristic peak of iron-sulfur bonds in the heme of CytC was observed at a wavelength of $699 \mathrm{~nm}$. The background spectrum was subtracted from the spectrum of CytC as it is shown in Fig. 6A according to the following equation:

$A_{F e-S}=A_{699}-\left(A_{670}+A_{728}\right) / 2$

It is based on the fact that the wavelength of the peak absorption of iron-sulfur bonds of $699 \mathrm{~nm}$ is exactly in the middle between the wavelengths of $670 \mathrm{~nm}$ and $728 \mathrm{~nm}$.

The series of consecutive spectra of Cyt-CL complex in different media was taken as following. First, the spectrum of $200 \mu \mathrm{M}$ aqueous solution of CytC was recorded. Then, $280 \mu 1$ of methanol and $70 \mu 1$ of $50 \mathrm{mM}$ TOCL were consequently added to $350 \mu \mathrm{l}$ of $200 \mu \mathrm{M}$ aqueous solution of CytC. The mixture was stirred by the vortexing with TTS2 (IKA Werke, Germany) at $1200 \mathrm{rpm}$ for $1 \mathrm{~min}$ after addition of each component. $50 \mu \mathrm{l}$ of the mixture was taken (for spectral analyses). $650 \mu \mathrm{l}$ of 
chloroform was added to the remaining solution following vortexing at $1400 \mathrm{rpm}$ for 3 minutes and centrifugation at $11700 \mathrm{~g}$ for $2 \mathrm{~min}$. Finally, $500 \mu \mathrm{l}$ of the lower fraction was taken up with the Pasteur pipette for spectrophotometric analysis.

\subsubsection{Analysis of Trp and Tyr fluorescence in CytC}

The measurements of absorbance were carried out using spectrofluorimeter RF-5421 PC (Shimadzu, Japan) in quartz cuvettes with an optical path length of $5.0 \mathrm{~mm}$ in a volume of the analyzed solution of $400 \mu \mathrm{l} .20 \mu \mathrm{M} \mathrm{CytC}$ was diluted twice either with water or with methanol and the fluorescence at the excitation wavelength of $289 \mathrm{~nm}$ in the range of 300-400 nm region was recorded. The Raman scattering of water appeared in the spectra was subtracted.

The fluorescence of Cyt-CL complex in a non-polar environment was measured in either chloroform-methanol mixture or $n$-hexane. It was found that chloroform because of its absorbency in UV is inappropriate for fluorescence measurement in UV region, and we gently evaporated the methanol-chloroform solution of Cyt-CL in a stream of nitrogen until wet pellet was obtained and re-dissolved the sample in $400 \mu$ of $n$-hexane.

\subsubsection{Chemiluminescence assay of Cyt-CL enzymatic activity}

For chemiluminescence analysis, Cyt-CL complex in chloroform-methanol media was prepared according to the following procedure. $100 \mu \mathrm{l}$ of $8.3 \mathrm{mM}$ TOCL in methanol was mixed with $233 \mu 1$ of $1.5 \mathrm{mM}$ aqueous CytC and $157 \mu \mathrm{l}$ of methanol (TOCL:CytC molar ratio is 2,37:1, water:methanol ratio is $10: 11$ by volume). Then, $490 \mu \mathrm{l}$ of chloroform was added and the obtained mixture was vortexed and centrifuged at $1350 \mathrm{~g}$ for $15 \mathrm{~min}$. Chloroform-methanol fraction containing the extracted Cyt-CL complex was taken up with the Pasteur pipette for the analysis.

Chemiluminescence was measured using Lum-5773 luminometer (DiSoft, Russia) operated with the software PowerGraph (developed by Izmailov D, MSU). Quasi-lipoxygenase activity of Cyt-CL complex was measured as follows. Background chemiluminescence of $21 \mu \mathrm{l}$ of $3.6 \mathrm{mM} \mathrm{BCL}$ (final concentration $150 \mu \mathrm{M}$ ) in methanol in the glass cuvette was registered for $30 \mathrm{sec}$, then the mixture of $13 \mu \mathrm{l}$ of $1 \mathrm{mM}$ C-525 (in methanol, final concentration $25 \mu \mathrm{M}$ ), $35 \mu \mathrm{l}$ of Cyt-CL complex in chloroform-methanol (prepared as described above) and $426 \mu \mathrm{l}$ of methanol was injected by syringe during continuous measurement of the chemiluminescence. The chemiluminescence kinetics of quasi-lipoxygenase process was recorded for $15 \mathrm{~min}$. To register the kinetics of lipoperoxidase reaction, the mixture of reagents from the first cuvette was injected by syringe to $5 \mu \mathrm{L}$ of $9 \mathrm{mM}$ hydrogen peroxide (90 $\mu \mathrm{M}$ final concentration) during continuous measurement of the chemiluminescence.

\section{Results}

\subsection{Size distribution of Cyt-CL nanoparticles}

\subsubsection{CytC in aqueous solution}

To check the possibility of determination of the mean size of approximately spherical nanoparticles of small size by using DLS method, we determined the mean hydrodynamic diameter of CytC in water solution (Fig. 1). It was found to be $3.6 \pm 1.0 \mathrm{~nm}$, which is close to the size of CytC in protein crystals or in aqueous solutions measured by X-ray diffraction or small-angle scattering methods 
(Vladimirov et al., 2011). As expected, the mean diameter of particles which was measured subsequently many times (Inset in Fig. 1) varied insignificantly that is typical for uniform particles like protein molecules.

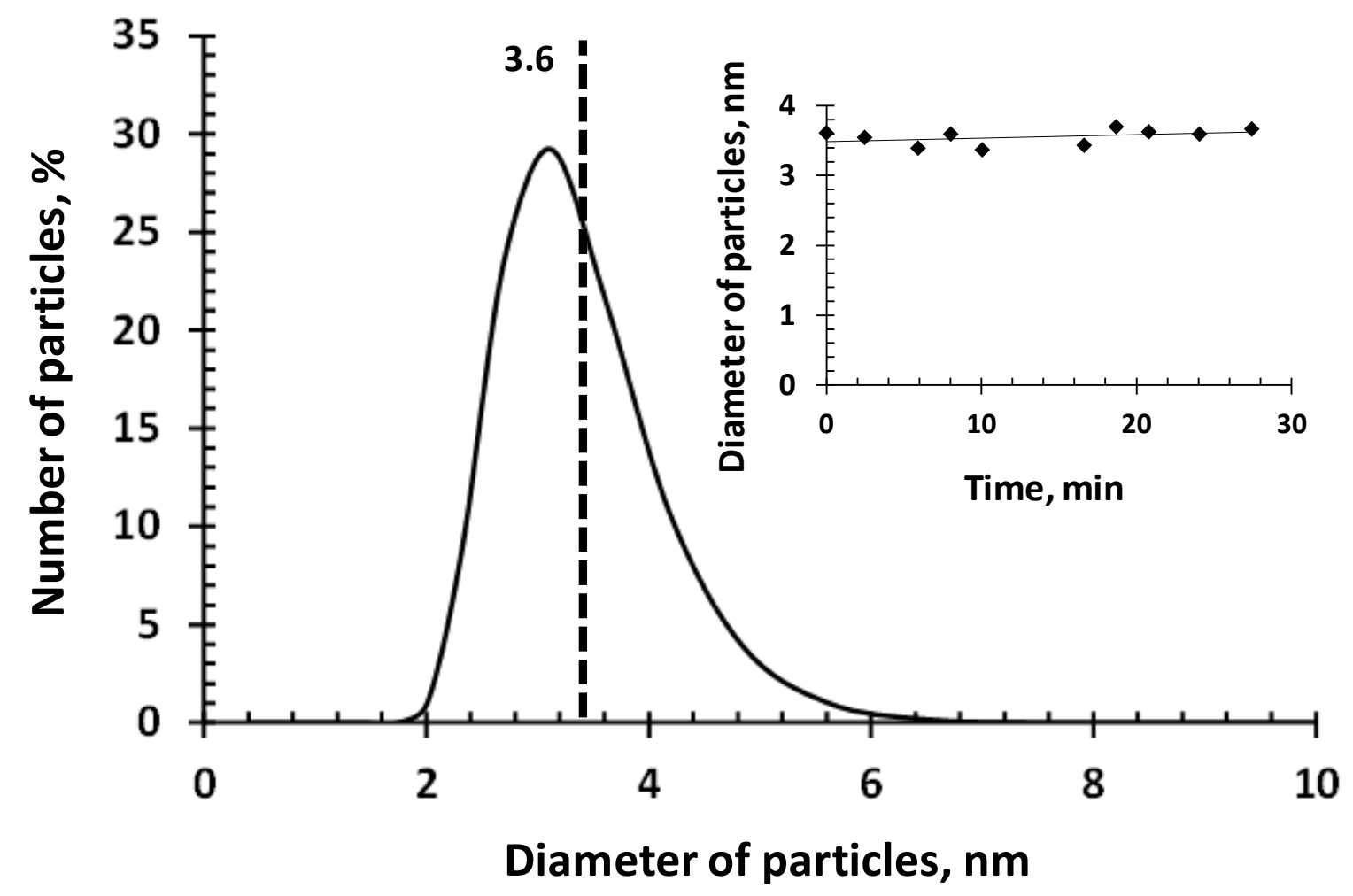

Figure 1. Size distribution by number of CytC in aqueous solution obtained by DLS measurements. The modal value for particle diameter $(\mathrm{nm})$ is shown by vertical dashed line. CytC concentration is $325 \mu \mathrm{M}$. Inset: Mean diameter of particles measured subsequently in the sample.

But we saw a completely different picture when we measured the chloroform-methanol solution of the Cyt-CL complex (Fig. 2).

\subsubsection{Cyt-CL in non-polar environnement}

We used Cyt-CL particles assembled from CytC and TOCL (1:30 mole/mole) in aqueous-methanol solution and then partitioned to chloroform, and find out that the mean diameter is $10.9 \pm 0.9 \mathrm{~nm}$ that is virtually equal to the diameter of Cyt-CL nanoparticles in Cyt-CL microcrystals $11 \pm 1 \mathrm{~nm}$ (Vladimirov et al., 2011). Unlike the $\mathrm{CytC}$ in aqueous solution, subsequently measured mean diameter of particles shows a strong fluctuation (Fig. 2, inset) that may be a result of variations of particles' size due to binding of different number of CL molecules on CytC surface of each given particle. 


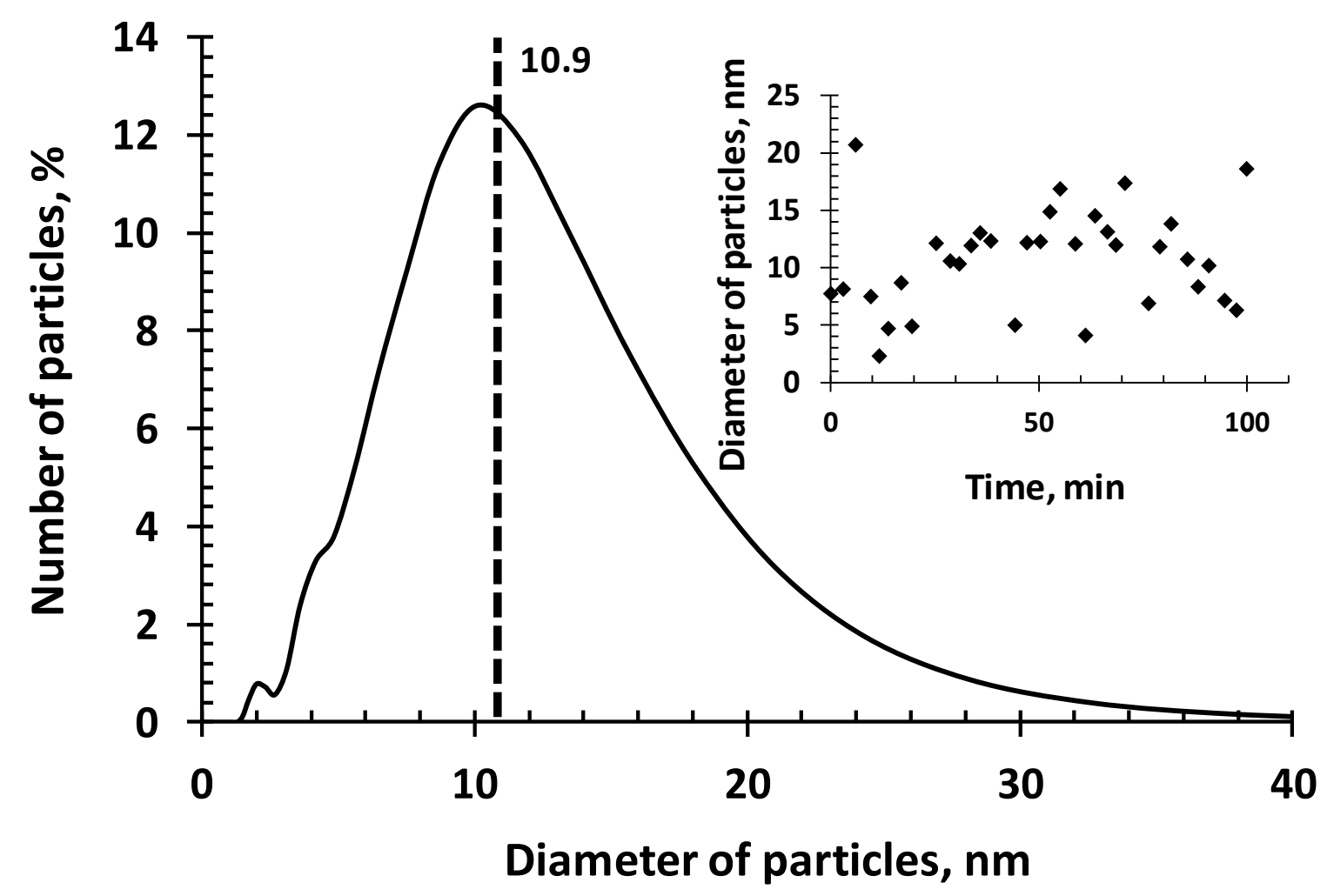

Figure 2. Size distribution by number of Cyt-CL in chloroform-methanol solution obtained by DLS measurements. The modal value for particle diameter $(\mathrm{nm})$ is shown by vertical dashed line. Inset: Mean diameter of particles measured subsequently in the sample.

Despite the limited ability of the DLS method to estimate particle sizes of different particles in the mixture, we still attempted to determine the Cyt-CL particles' size distribution using a histogram (Fig. 3A), where the number of particles lying in a certain size range (1.5 nm in Fig. 3A) was plotted as a function of the average particle size in each range of diameters. Three dominant sizes could be revealed 4.0 - 5.5, 7.0 - 8.5, and 11.5 - $13.0 \mathrm{~nm}$ (Groups 1-3). The number of measurements showing these parameters was, correspondingly, 4, 5 and 8 (Fig. 3A). Fig. 3B shows the distribution of mean sizes of Cyt-CL inside these small-sample groups. The average size of the particles within the groups 1,2 , and 3 has been calculated to be $4.7 \pm 0.7,7.8 \pm 1.0$, and $12.1 \pm 1.4 \mathrm{~nm}$, respectively. 


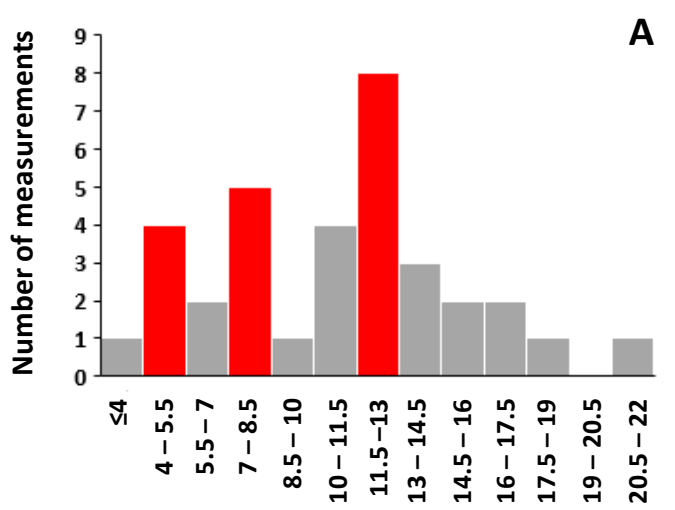

Mean diameter of particles, $\mathrm{nm}$

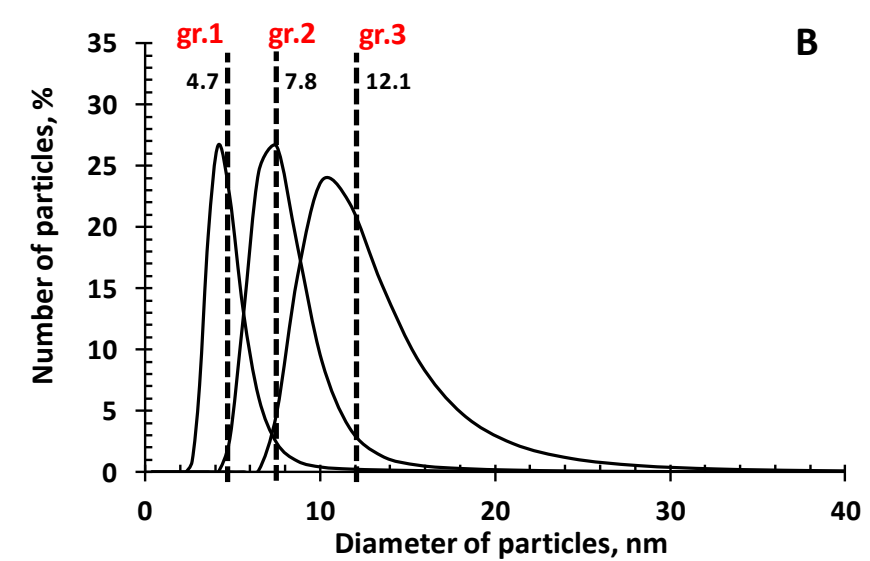

Figure 3. Distribution of mean particle sizes as a function of a number DLS measurements for CytCL complex assembled in non-polar environment (methanol-chloroform mixture) at CytC and CL concentrations in water-methanol solution of $162.5 \mu \mathrm{M}$ and $5 \mathrm{mM}$, respectively.

If the distribution of the nanoparticles by sizes really involves grouping around three dominant diameters, it is tempting to compare these data with those obtained by the SAXS method. It is striking that the particle sizes coincide in these two systems: $7.8 \pm 1.0$ in Fig. $3 \mathrm{~B}$ versus $8.0 \pm 0.7 \mathrm{~nm}$ in microcrystal sediment (Vladimirov et al., 2013a) and $12.1 \pm 1.4$ in Fig. 3B versus $11.1 \pm 1.0 \mathrm{~nm}$ in microcrystals (Vladimirov et al., 2011). The compared pairs of values are the same within the measurement error. The nature of the smallest particles of Cyt-CL in the chloroform solution is now a matter of speculations (See Discussion for more detail).

Before transferring the complex of Cyt-CL to the hydrophobic phase, we mixed the aqueous solution of cytochrome $\mathrm{c}$ with the cardiolipin solution in methanol (see Section 2.2.1). We measured the particle size of the complex formed in this mixture, i.e. complex Cyt-CL in 50\% methanol (Fig. 4, blue line). We also measured a solution of the cardiolipin alone in 50\% methanol (Figure 4, red line) and a solution of cardiolipin in the chloroform-methanol hydrophobic phase after phase separation in a system containing cardiolipin but not containing cytochrome (Figure 4, black line).

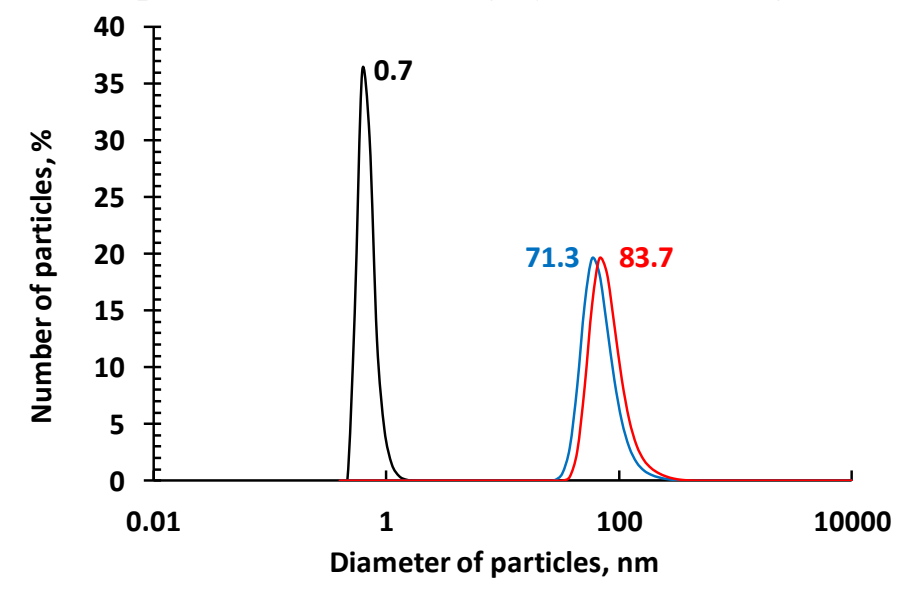

Figure 4. Distribution of mean particle sizes as a function of a number DLS measurements. Left peak (black) - TOCL molecules in chloroform-methanol phase after phase separation of watermethanol-chloroform mixture containing CL without CytC. Right peaks - nanoparticles in watermethanol solution before adding chloroform. Blue $-\mathrm{CytC}+\mathrm{CL}$, Red $-\mathrm{CL}$ alone. CytC and CL concentrations are $162.5 \mu \mathrm{M}$ and $6.75 \mathrm{mM}$, respectively. 
As expected, CL in hydrophobic solvent showed uniform particles of $0.7 \pm 0.1 \mathrm{~nm}$ in diameter, which individual molecules may have (Fig. 4, black line). In 50\% methanol, CL behave as rather uniform particles of $83.7 \pm 9.8 \mathrm{~nm}$, which corresponds to a very small unilamellar liposomes of maximal possible membrane curvature and minimal possible size (Fig. 4, red line). Notably, that Cyt-CL complex in $50 \%$ methanol had practically the same size $71.3 \pm 11.6 \mathrm{~nm}$. Unfortunately, due to peculiarity of the method (the light scattering depends on 6 power of the diameter), we cannot distinguish two cases: (1) cytochrome $c$ is not associated with cardiolipin under these conditions, or (2) cytochrome $c$ binds to liposome membranes without causing a significant change in their size.

\subsection{Conformation of CytC in the complex of Cyt-CL in a non-polar environment}

As mentioned above, CytC does not possess significant fluorescence in an aqueous solution, but if the protein globule is partially melted, the fluorescence of Tyr and Trp residues appears (Belikova et al., 2006; Kapralov et al., 2011). Figure 5A presents the fluorescence spectra of Cyt-CL in nhexane at different excitation wavelengths. Under excitation by UV at 268 and $275 \mathrm{~nm}$, the fluorescence of both Tyr residues (maximum at $307-310 \mathrm{~nm}$ ) and Trp residues (the maximum at $330 \mathrm{~nm}$ ) has been registered. At longer excitation wavelengths (282 and $289 \mathrm{~nm}$ ) one can see mainly the fluorescence of Trp residues, with the maximum at $330 \mathrm{~nm}$ and two shoulders at about 315 and $340 \mathrm{~nm}$ (Fig. 5A). This is typical for indole ring fluorescence in hydrophobic environment (Konev, 1967). Unfortunately, we failed to convert cytochrome c from the aqueous solution to heptane and measure its fluorescence for comparison with the fluorescence of the Cyt-CL complex, but we compared our spectra with the fluorescence spectra of tyrosine and tryptophan residues derived from data (Kapralov et al., 2011 1) (dashed lines in Figure 5A). The maximum of tryptophan in the complex lies in the same region as in our conditions, but does not have a pronounced vibrational structure.
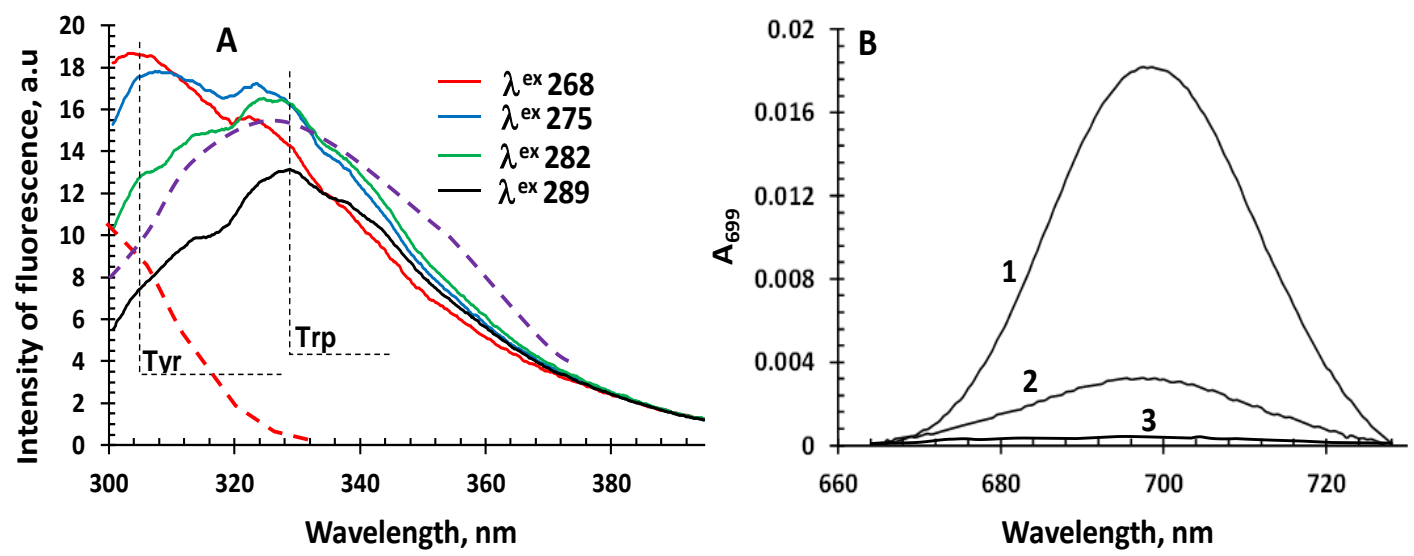

Figure 5. (A) - Fluorescence spectra of Cyt-CL complex in hexane at different excitation wavelengths. (B) - Absorption of $50 \mu \mathrm{M}$ and $12.8 \mu \mathrm{M}$ CytC in water, (1) and (2), respectively, and of $9.8 \mu \mathrm{M}$ Cyt-CL complex in hexane (3); absorption is due to Fe $\cdots>\mathrm{S}$ (Met80) bond. Cyt-CL complex formed in non-polar solvent (methanol-chloroform mixture) and replaced to hexane. For comparison we placed the fluorescence spectra (dashed lines) of Tyr and Try residues in the complex of Cyt $\mathrm{C}$ with CL in aqueous solutions at the excitation at $275 \mathrm{~nm}$ (From (Kapralov et al., 2011)). 
As it can be concluded from Fig. 5B, melting of the CytC globule in the Cyt-CL complex formed in methanol-chloroform $(1: 1 \mathrm{v} / \mathrm{v})$ medium is accompanied by the disappearance of the absorption band (at $699 \mathrm{~nm}$ ) of the $>\mathrm{Fe} \cdot \mathrm{S}$ (Met80) coordination bond. Thus, the conformation of CytC in the complex changes and the complex may acquires enzymatic activity of quasi-lipoxygenase and lipoperoxidase (Vladimirov et al., 2017).

\subsection{Effect of methanol on the conformation of $\mathrm{CytC}$}

In the previous section, we used two criteria to judge the unfolding of the cytochrome c protein globule in Cyt-CL: the disappearance of the absorption band at $699 \mathrm{~nm}$ and the appearance of fluorescence of tyrosine and tryptophan residues. These criteria had been used by other authors in model experiments with CytC dissolved in methanol (and other alcohols) mixed with water (Wei and Danielson, 2011) (Jain et al., 2013). They found that the protein unfolding, determined by a number of methods, occures in increased concentrations of CytC from 45 to $60 \%$ v/v (Jain et al., 2013) or from 40 to $70 \% \mathrm{v} / \mathrm{v}$ (Wei and Danielson, 2011), accompanied with the increase of fluorescence excited by $280 \mathrm{~nm}$ and emitted at 320-400 nm (Jain et al., 2013) or at $340 \mathrm{~nm}$ (Wei and Danielson, 2011). Only fluorescence intensities are shown in the cited papers, rather than fluorescence spectra, and light absorbency of $>\mathrm{Fe} \cdot \cdot \mathrm{S}($ Met80) coordination bond (695-700nm) that is broken in unfolded state of CytC had also not been measured. On our experiments we compared the data obtained from CytC in water/methanol mixtures with those of Cyt-CL dissolved in hydrophobic solvents by using spectrofluorometry and spectrophotometry at approximately equal concentrations of CytC, temperatures, and other conditions.
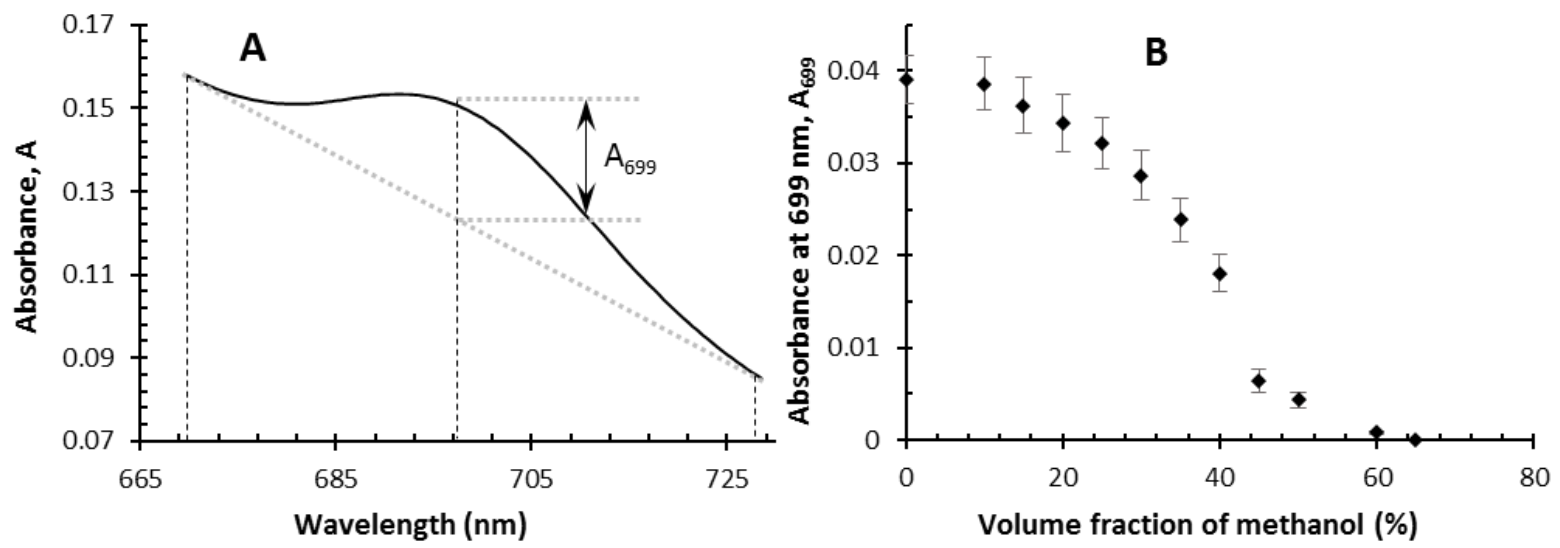

Figure 6. (A) - Absorbance spectrum of $\mathrm{CytC}$ in water solution. (B) - The absorption of $200 \mu \mathrm{M}$ CytC solution at $699 \mathrm{~nm}$ plotted as a function of the methanol volume fraction in the mixture.

Figure 6B shows the effect of the volume fraction of methanol in water-methanol mixture on the absorption of CytC at $699 \mathrm{~nm}$. This wavelength which corresponds to the maximum of absorption of the non heme iron/sulfur coordination bond $>\mathrm{Fe} \cdot \mathrm{S}(\mathrm{Met} 80)$, estimated as shown in Fig. 6A.

At the same time, the volume of the protein globule in $50 \%$ aqueous-methanol solution increases as evidenced by the appearance of fluorescence of Trp residues (Fig. 7A). It is of note that the maximum of fluorescence lies here not at $330 \mathrm{~nm}$ (as in solutions of most Trp-containing proteins and for Cyt$\mathrm{CL}$ in hydrophobic environment, Fig.5A) but closer to $350 \mathrm{~nm}$, which is typical for aqueous environment of Trp (Burshtein et al., 1966; Vladimirov and Zimina, 1965). 

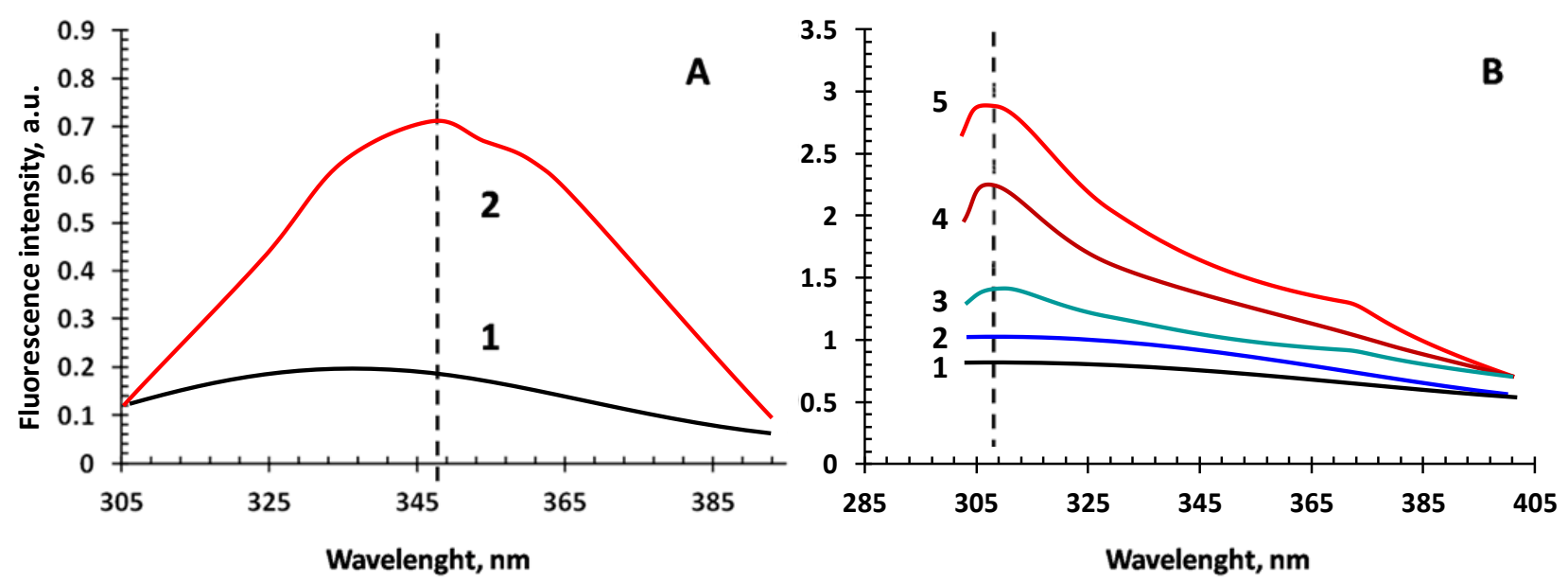

Figure 7. Fluorescence spectra of CytC in water-methanol solutions. A. Trp residue fluorescence. $10 \mu \mathrm{M}$ CytC in water (1) and water-methanol solution, $50 \% \mathrm{v} / \mathrm{v}$ (2). Excitation wavelength is 289 $\mathrm{nm}$. The peak in (2) at about $350 \mathrm{~nm}$ corresponds to fluorescence of Trp residue. B. Tyr residue fluorescence. $10 \mu \mathrm{M} \mathrm{CytC}$ in water-methanol solutions with different methanol/water volume ratio: (1) $0 \%$, , (2) $20 \%$, (3) $30 \%$, (4) $40 \%$, (5) $50 \%$ v/v. Excitation wavelength is $268 \mathrm{~nm}$. The peak at about $307 \mathrm{~nm}$ corresponds to fluorescence of Tyr residue.

Fig. 7B shows that Tyr residue fluorescence, virtually absent in water (Curve 1) increases with growing concentration of methanol. This implies unfolding of the protein and increase the distance between Tyrs and heme, as well as in case of Try residue (Fig. 7A).

\subsection{Enzymatic activity of Cyt-CL in non-polar environment}

The oxidation of lipids in the mitochondrial membrane is mediated by the reactions of lipid radicals $\mathrm{L} \cdot$ and $\mathrm{LOO} \bullet$ which are formed by the complex of CytC with anionic lipids, predominantly with mitochondrial CL. The interaction of two LOO radicals is accompanied by chemiluminescence (Vladimirov, 1996) which can be detected in the presence of physical enhancer coumarin (C-525) (Vladimirov et al., 1995) and thus can be a measure of the enzymatic activity of the Cyt-CL complex. The lipoperoxyl radicals can be formed by two different reactions: i) radical decomposition of preformed lipid hydroperoxides $(\mathrm{LOOH})$, is known as quasi-lipoxygenase reaction and ii) the peroxidation of lipids in the presence of oxidants (e.g. $\mathrm{H}_{2} \mathrm{O}_{2}$ ) - lipoperoxidase reaction. Cyt-CL complex has both quasi-lipoxygenase and lipoperoxidase activity in aqueous media (Vladimirov et al., 2017). In this study, enzymatic activity of Cyt-CL complex in the non-polar environment has been investigated.

The naturally occurring BCL is composed mainly from unsaturated tetra-linoleoyl cardiolipin which is prone to oxidation, in this study it was used with a dual purpose: as a source of preformed lipid hydroperoxides (LOOH) for quasi-lipoxygenase reaction and as a oxidisable substrate for lipoperoxidase reaction. 

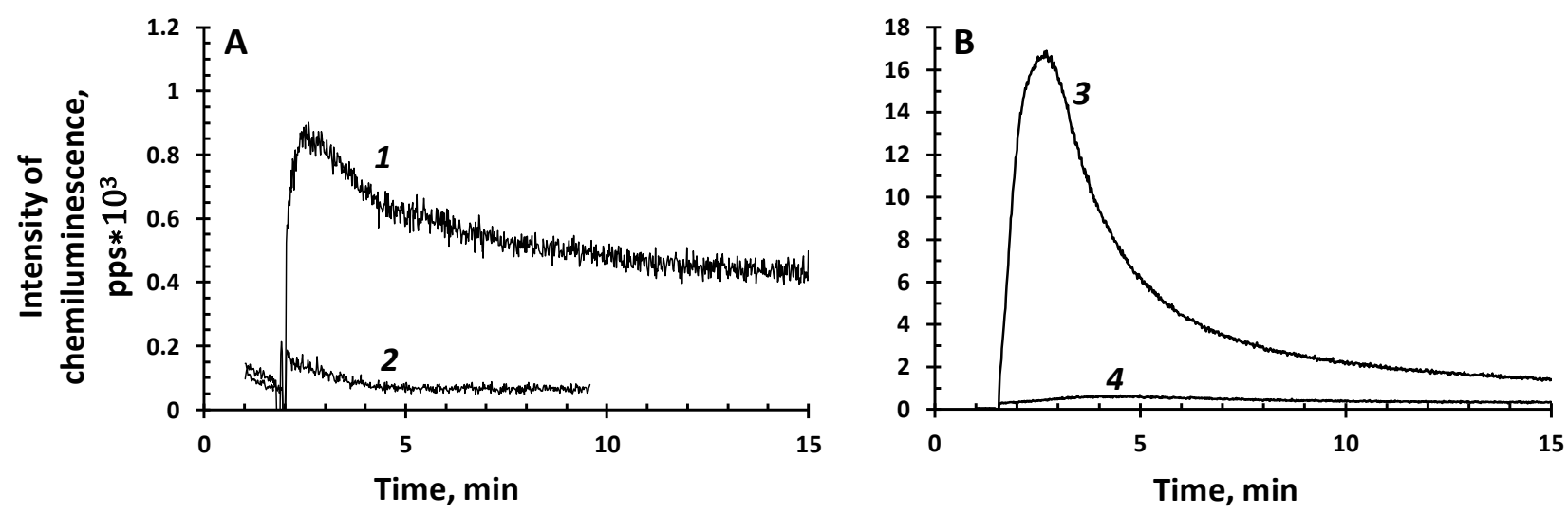

Figure 8. (A) - Lypoxygenase activity of Cyt-CL in chloroform-methanol medium. Time phase 1 (0-1.2 min) corresponds to BCL (1) or methanol (2) placed into a cuvette. Phase 2 (1.2-9 min) corresponds to addition of Cyt-CL and C-525 in chloroform-methanol medium. Resulting concentrations at the start of the lypoxygenase reaction (at $1.2 \mathrm{~min}$ ): $\mathrm{CytC}-5 \mu \mathrm{M}$ (estimated by spectrophotometry), BCL - $150 \mu \mathrm{M}, \mathrm{C}-525-25 \mu \mathrm{M}$. (B) - Lypoperoxidase activity of Cyt-CL in chloroform-methanol medium. Phase 1 (0-1.2 min) corresponds to addition of $\mathrm{H}_{2} \mathrm{O}_{2}(3)$ or methanol (4) in a cuvette. Phase 2 (1.2-9 min) - addition of the solution from the probe in A. Resulting concentration of $\mathrm{H}_{2} \mathrm{O}_{2}$ at the start of the reaction - $90 \mu \mathrm{M}$.

Fig. 8A represents chemiluminescence kinetics of the quasi-lipoxygenase process induced by the addition of Cyt-CL complex in chloroform-methanol media to BCL in the presence of coumarin C525 (curve 1). The addition of the complex to methanol instead of BCL did not show any chemiluminescence (Fig. 8A, 2), the exclusion of other components of Cyt-CL system (coumarin or $\mathrm{CytC}$ ) lowered the chemiluminescence signal to the background level (data not shown).

When preformed in BCL lipid hydroperoxides were completely decomposed, hydrogen peroxide was added to the same probe. This induced the formation of a new portion of lipid radicals which has been accompanied by a rise of chemiluminescence (Fig. 8B, 3). In this reaction, lipoperoxyl radicals (LOO•) are formed by the oxidation of lipid molecules (LH) by $\mathrm{H}_{2} \mathrm{O}_{2}$ in the presence of Cyt$\mathrm{CL}$ which acts as a lipoperoxidase. In the absence of $\mathrm{H}_{2} \mathrm{O}_{2}$ the lipoperoxidase process does not take place (Fig. 8B, 4).

\section{Discussion}

\subsection{Cyt-CL complex feature in a hydrophobic solvent}

We showed earlier in the SAXS study of the sediment of the CytC complex with TOCL that the complex had a microcrystalline structure with interplanar reflection distances of 8.0 and $11.1 \mathrm{~nm}$ (Proskurnina et al., 2013; Shtykova et al., 2013; Vladimirov et al., 2013a; Vladimirov et al., 2011). This allowed us to assume that the complex formed in nature is a nanosphere of approximately the same diameter, consisting of a CytC molecule and a monolayer of CL surrounding this molecule. In the present paper we showed (Fig. 3) that in a hydrophobic solvent the Cyt-CL complex presents as two main groups with characteristic mean diameters of $7.8 \pm 1.0$ and $12.1 \pm 1.4 \mathrm{~nm}$ (Fig. 3). In addition, the minor fractions of the particles of smaller $(4.7 \pm 0.7 \mathrm{~nm})$ and larger sizes (above $16 \mathrm{~nm})$ have also been registered. This is schematically summarized in Fig. 9A. 


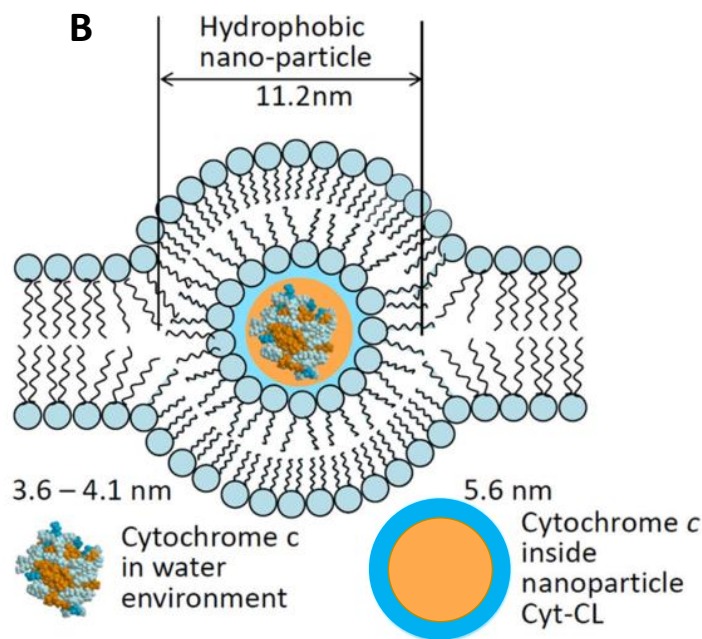

Figure 9. Schematics showing hypothesized structure of Cyt-CL complex self-assembled in nonpolar solvent (A) and in the lipid bilayer of mitochondrial membrane (B). A - Average sizes of nanospheres of CytC and the Cyt-CL complex have been obtained by DLS in this work (Fig. 1 and 2). Red and orange circles are $\mathrm{CytC}$ molecules in a native (compact) and unfolded (melted) conformation, respectively. Violet circle is a partially unfolded $\mathrm{CytC}$ with hydrophobic groups on its surface. Blue circles are lipid layers of CL molecules surrounding CytC. B - The hypothesis of the incorporation of Cyt-CL nanosphere into the lipid bilayer of mitochondrial membrane first proposed in (Vladimirov et al., 2013b), the sketch is adopted from (Marchenkova et al., 2015). The diameter of CytC core inside the complex $(5.6 \mathrm{~nm})$ was calculated from SAXS data obtained using microcrystalline precipitate of Cyt-CL. A thin blue border around the light brown core (B, bottom right) is the polar surface of the molten globule of CytC.

It is very important that the diameters of two major groups of particles $(12.1 \pm 1.4 \mathrm{~nm}$ and $7.8 \pm 1.0$ $\mathrm{nm}$ ) is rather close to $11.2 \mathrm{~nm}$ obtained in microcrystalline sediment of Cyt-CL (Vladimirov et al., 2011) and $11.1 \pm 1.0 \mathrm{~nm}$ and $8.0 \pm 0.7 \mathrm{~nm}$ obtained in our more recent experiments with Cyt-CL sediments (Shtykova et al., 2013; Vladimirov et al., 2013a). The cardiolipin-to-cytochrome c ratio evaluated by centrifugation and spectrophotometry was found to be constant at each given situation (Vladimirov et al., 2011), the lipid to protein molar ratio being different at different $\mathrm{pH}: 13 \pm 4$ at $\mathrm{pH}$

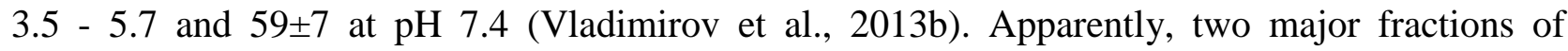
nanoparticles observed by DLS in this work are different in size because of different number of cardiolipin molecules in the lipid monolayer covering the protein globule. It is easy to calculate the approximate volume of the lipid monolayer $\left(\mathrm{V}_{\mathrm{L}}\right)$ in a nanosphere with the diameter $8 \mathrm{~nm}$ or $12 \mathrm{~nm}$, if the radius of the protein globule inside the nanosphere is $5,6 \mathrm{~nm}$. The calculated ratio of $\mathrm{V}_{\mathrm{L}}(12) / \mathrm{V}_{\mathrm{L}}(8)=4.62$. The ratio of the number of lipid molecules (n) in the Cyt-CL particles of these two types $n(12) / n(8)=59 / 13=4.54$. We may conclude that the difference in the size of two major fractions of Cyt-CL (lipid layer thickness 2,8 and 1,0 $\mathrm{nm}$ for larger and smaller particles) depends on the amount of the lipid molecules attached to the cytochrome c surface, rather than on the difference of the protein conformation in the nanospheres.

Interpretation of DLS data for two minor fractions of nanoparticles ( 4.7 and $\geq 16 \mathrm{~nm}$ in Fig. 3 and Fig. 9A) is not so unambiguous. Probably we may attribute the $4.7 \mathrm{~nm}$ particles to $\mathrm{CytC}$ with changed conformation and hydrophobic groups exposed on its surface. The data on the conformation of 
cytochrome at the water surface ("stratified" CytC) may testify in favor of this assumption (Marchenkova et al., 2015), although some additional data are needed. As for particles of $16 \mathrm{~nm}$ or more, we found nothing better than to consider them aggregates of nanoparticles (Fig. 9A, left). These particles may be formed by a different number of nanospheres, and a partial fusion of lipid monolayers may occur on the surface of the complex.

\subsection{Cytochrome c conformation in Cyt-CL nanospheres}

The data obtained in this work showed that cytochrome $c$ inside the Cyt-CL nanosphere immerged in the hydrophobic solvent has a partially molten conformation (Fig. 5). Earlier data on the Cyt-CL sediments showed that the size of CytC inside nanosphere is essentially larger than that of CytC dissolved in water (Vladimirov et al., 2011), this is also valid for Cyt-CL dissolved in chloroform (Fig. 1 - 3) so showing the molten state of the $\mathrm{CytC}$ globule. In the presence of cardiolipin-containing liposomes, $\mathrm{CytC}$ acquires the ability to fluoresce in the ultraviolet region of the spectrum due to the residues of tyrosine and tryptophan (Belikova et al., 2006 10; Kapralov et al., 2011). In our work, strong tyrosine and tryptophan fluorescence was observed in Cyt-CL dissolved in hexane (Fig. 5). In both cases, the data indicate an increase of the distance between Tyr and Trp residues and heme, the energy acceptor, which in native cytochrome $c$ is less than the Förster radius for these donoracceptor pairs. Fig. 5 shows that the fluorescence belongs to tryptophan only under excitation at 289 $\mathrm{nm}$, since tyrosine does not absorb at this wavelength (Fig.5A), while tyrosine fluorescence maximum at $305 \mathrm{~nm}$ dominate at the excitation at $265 \mathrm{~nm}$. Note that the tryptophan spectrum shows fine structure and maximum characteristic of indole ring in hydrophobic environment (Konev, 1967; Vladimirov, 1969). At the same time, a weak absorption band of about $700 \mathrm{~nm}$ disappears in this object (Fig. 5B), which indicates the disruption of the coordination bond between the heme iron and sulfur atom of the amino acid residue Met80 (Belikova et al., 2006; Vladimirov et al., 2006b). This also supports the conclusion that in Cyt-CL nanoparticles dispersed in hydrophobic medium CytC has molten conformation with $>\mathrm{Fe} \cdots \mathrm{S}($ Met80) bonds broken that implies the peroxidase activity of the hemoprotein. And indeed, the Cyt-CL complex was shown to have the pronounced enzymatic activity as quasi-lipoxygenase and lipoperoxidase in chloroform solution (Fig. 8).

Changes in the conformation of cytochrome $\mathrm{c}$ within the nanosphere in comparison with the aqueous solution of hemoprotein in all indices (fluorescence of Tyr and Trp residues, absorption at $670 \mathrm{~nm}$ ) is similar to that of cytochrome c in 50\% methanol (compare Figures 5-7). Such a change in the conformation is called partial melting or unfolding (Belikova et al., 2006 10) and it improves the availability of heme for hydrogen peroxide (Vladimirov et al., 2006a 9). Partial unfolding of the protein leads to the appearance of peroxidase activity due to the disruption of the coordination bond between heme iron and sulfur of Met80 (Belikova et al., 2006 10).

As mentioned in the Introduction, the change in CytC conformation after its binding with cardiolipin was mentioned almost by all researches involved. The conformation of the protein in reverse micelles of cardiolipin in hydrophobic environment had not been studied before. A related system, with $\mathrm{CytC}$ encased in a reversed micelle suspended in an organic solvent, was studied by NMR recently in Wand's laboratory (O'Brien et al., 2015). They showed that the encapsulation of oxidized horse cytochrome $\mathrm{c}$ in 1-decanoylrac-glycerol/lauryldimethylamine-N-oxide (LDAO)/hexanol reverse micelles prepared in pentane yields NMR spectra essentially identical to the protein in free 
aqueous solution. Obviously, the protein was not significantly disturbed by the binding of cardiolipin in the context of the reverse micelle. Apparently, cardiolipin molecules in the lipid shell of nanoparticles was attached more specifically and stronger to the charged groups on protein core than LDAO. The nature of the lipid structure is very essential for is affinity to the CytC (Stepanov et al., 2009).

In the literature, there are diverse interpretations of data about the protein conformation in membrane-bound Cyt-CL. Mandal et al. have found that membrane binding results in CytC gaining the increased peroxidase activity, but they do not observe evidence for large-scale unfolding or penetration into the membrane core (Mandal et al., 2015). In this paper, the increase of peroxidaze activity of bound CytC increased only 2 times as compared with $\mathrm{CytC}$ without lipid. At higher $\mathrm{CL} / \mathrm{CytC}$ ratio (25/1 and more) the peroxidase acvivity of CytC bound to CL-containing liposomes was about 30 times higher than that of CytC alone (Belikova et al., 2006); the protein unfolding was rather strong in this case, as evidenced by tryptophan and tyrosin fluorescence increase (Belikova et al., 2006; Kapralov et al., 2007). Docking studies with linoleic acid, the most common fatty acid component of cardiolipin, show that $\mathrm{C} 11$ of linoleic acid can sit adjacent to Tyr67 and the heme, consistent with the oxygenation pattern observed in lipidomics studies (McClelland et al., 2016). By measuring energy transfer from the fluorophore to heme in dancyl (Dns)-labeled variants of horse heart cyt c, a conformational diversity of the CL-bound CytC ensemble with distinct populations of the polypeptide structures that vary in their degree of protein unfolding (Hanske et al., 2012). This and other data (See (Pandiscia and Schweitzer-Stenner, 2015), for example) were interpreted in terms of CytC binding to the outer surface of a lipid bilayer, but they did not consider at all the alternative possibility, the formation of hydrophobic Cyt-CL nanospheres, in which CytC is in a partially unfolded state (Vladimirov et al., 2013a; Vladimirov et al., 2011; Vladimirov et al., 2013b). Meanwhile, de Kruijff and Cullis, in experiments with liposomes composed of cardiolipin showed by 31P-NMR and freeze-fracture techniques that CytC specifically induces the hexagonal H\| phase and possibly an inverted micelle structure (de Kruijff and Cullis, 1980). They hypothesized that a nanosphere, very similar to that shown in Fig. 9B of this paper, may be formed inside the membrane lipid bilayer in the course of $\mathrm{CytC}$ transport across the membrane. In reality, CytC alone cannot penetrate through the lipid bilayer, but it can do so in the complex with cardiolipin, the fact supported for artificial lipid membranes in subsequent publications (Antonov et al., 2014; Bergstrom et al., 2013; Firsov et al., 2015).

\subsection{Reactions of free radical formation by $\mathrm{Cyt}-\mathrm{CL}$ in hydrophobic environment}

Unlike other hemoprotein in oxidized form $\left(\mathrm{Fe}^{3+}\right), \mathrm{CytC}$ does not catalyze the oxygenation of organic substrates by hydroperoxides. The Cyt-CL complex formed by mixing $\mathrm{CytC}$ with cardiolipin by using protocol like that used in this paper (Cardiolipin to CytC ratio was 32) is a rather potent peroxidase (Vladimirov et al., 2009b) and lipoperoxidase (Vladimirov et al., 2009a). The reaction mechanism of the processes may be illustrated by the scheme in Fig. 10. 


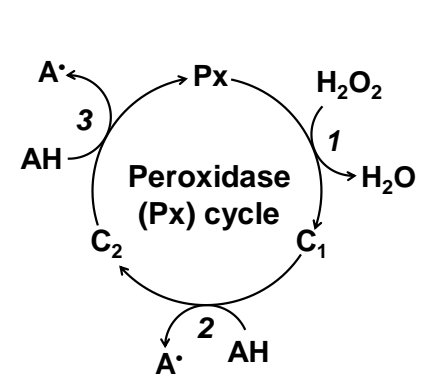

Peroxidase cycle
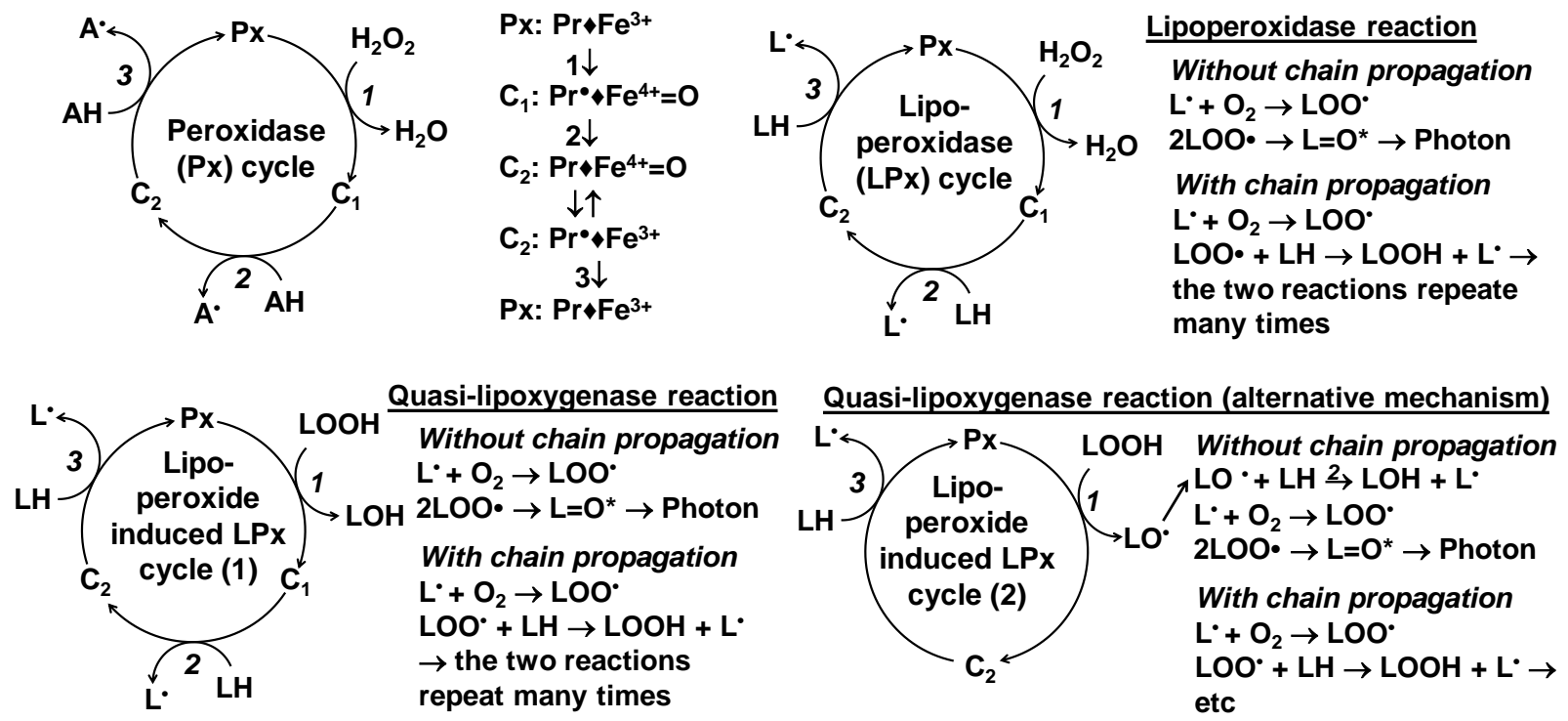

Figure 10. Proposed mechanisms of peroxidase and lipoperoxidase reactions catalyzed by Cyt-CL complex. Px - Peroxidase; $\mathrm{Pr}$ - apo-peroxidase; $\mathrm{Pr}^{\circ}$ - protein free radical; $\mathrm{C}_{1}$ - Complex I (Compound I); $\mathrm{C}_{2}-$ Complex II; $\downarrow$ - porphyrin ring of the peroxidase heme; $\mathrm{AH}$ - the reaction substrate $2 ; \mathrm{A}^{\bullet}$ the substrate free radical; $\mathrm{LH}$ - polyunsaturated fatty acid, the reaction substrate $2 ; \mathrm{L}^{\cdot}-$ lipid (alkyl) radical; $\mathrm{LOO}^{\circ}$ - lipid peroxyl radical; $\mathrm{L}=\mathrm{O}^{*}$ - a molecule of ketone, a product of disproportionation reaction of two LOO', in excited state. Top raw, left: Peroxidase cycle in reactions catalyzed by horse radish peroxidase (HRP), first studied by B. Chance by using rapid flow kinetic spectrophotometry (Chance, 1949, 1952; Chance and Higgins, 1952) (Cf. (Furtmuller et al., 2004)). Top raw, right: Lipoxyperoxidase cycle, polyunsaturated fatty acid chains of phospholipids (LH) are the substrate 2. Bottom raw,left: Lipoperoxidase reaction scheme in case LOOH is the substrate 1 . Bottom raw, right: A hypothetic scheme of the same reaction as in Bottom raw, left, except the reaction of $\mathrm{Px}$ with $\mathrm{LOOH}$ is a one-electron transition to form $\mathrm{LO}^{*}$ radical and $\mathrm{C}_{1}$ peroxidase intermediate. The final products of one cycle involve two lipid radicals $\mathrm{L}^{\bullet}$ in both cases.

In peroxidases the reaction cycle involves essentially 3 subsequent reactions (Chance, 1952)

$\mathrm{HPR}+\mathrm{ROOH} \rightleftarrows$ Complex I,

Complex I $+\mathrm{AH} \rightarrow$ Complex II $+\mathrm{A}$

Complex II + AH $\rightarrow \mathrm{HPR}+\mathrm{A}$,

where the hydroperoxide $\mathrm{ROOH}$ is the first, and $\mathrm{AH}$ - the second substrate of the reaction, while the primary reaction product, denoted as A (Chance, 1952), is in fact a free radical, $\mathrm{A}^{*}$. In experiments where luminol (LumH) is the second substrate, the formation of luminol radical (Lum') is accompanied by chemiluminescence (Cormier and Prichard, 1968) due to a series of consecutive reactions (see review (Vladimirov and Proskurnina, 2009)). Cyt-CL (in concentrations about 10 $\square \mathrm{M}$ ) in water solutions containing luminol shows the chemiluminescence burst upon addition of $\mathrm{H}_{2} \mathrm{O}_{2}$ or organic hydroperoxides (Belikova et al., 2006; Osipov et al., 2006; Vladimirov et al., 2006c), whereas flavonoid antioxidants inhibit the chemiluminescence at micromolar concentrations (Vladimirov et al., 2006c) probably because of scavenging the luminol radical. These data substantiate the conclusion that the reaction scheme of peroxidase cycle (Fig. 10 upper raw, left) is also valid for Cyt-CL in aqueous medium. 
The lipoperoxidation reaction catalyzed by Cyt-CL is of interest for cell biology, pathology (Tyurina et al., 2014) and death (Kagan et al., 2009a; Kagan et al., 2005). We showed earlier that Cyt-CL nanoparticles suspended in buffered water medium produce lipid peroxyl radicals upon addition $\mathrm{H}_{2} \mathrm{O}_{2}$ (Vladimirov et al., 2009a; Vladimirov et al., 2017) and that antioxidants inhibit the lipoperoxidase reaction (Vladimirov et al., 2017). These data show that the mechanism of lipid peroxidation catalyzed by Cyt-CL is the same as that in "classic peroxidases" (Fig. 8, upper row left and (Furtmuller et al., 2004)).

In the presence of oxygen, the primary product of lipoperoxidase reaction $\mathrm{L}^{\bullet}$ reacts immediately with $\mathrm{O}_{2}$ to form peroxyl radical LOO'. This radical is of great interest for the biology and pathology of the cell because initiates chain lipid peroxidation in cytoplasmic and mitochondrial membranes (Vladimirov et al., 1969; Vladimirov and Archakov, 1972; Vladimirov et al., 1980). Due to essential chain length (the ratio of initial radical concentration to the amount of hydroperoxides formed) (Cheremisina et al., 1972) the stoichiometric ratio of the amount of LOOH formed to the consumed $\mathrm{H}_{2} \mathrm{O}_{2}$ in mitochondrial membranes can reach one order of magnitude (see also (Shimada and Yasuda, 1977)).

\subsection{The hypothesis on the incorporation of the Cyt-CL complex into the mitochondrial membrane lipid bilayer}

The data obtained in this work about the sizes and enzymatic activity of Cyt-CL nanospheres are in a good agreement with the hypothesis that the nanospheres may exist and perform lipoperoxidase function inside the biological membrane bilayer (Fig. 9B). The hypothesis was first proposed in the review (Vladimirov et al., 2013b) and then discussed in respect to the properties of cardiolipin and CytC monolayers (Marchenkova et al., 2015). Because of the lack of direct evidence, we are still leaving aside the question of a detailed picture of the transformation of the membrane-bound CytC into a nanosphere. But then the main question is whether such a nanosphere can exist in a hydrophobic environment, how large is it, whether it catalyzes lipoperoxidase reactions, and what is the mechanism of these reactions. In the present work, it has been shown that hydrophobic Cyt-CL nanoparticles of size $12.1 \pm 0.7$ and $7.6 \pm 0.7 \mathrm{~nm}$ do exist in the hydrophobic environment, that the nanoparticles catalyze the reactions of the lipoperoxidase cycle to form lipoperoxyl radicals, with the first substrate being $\mathrm{H}_{2} \mathrm{O}_{2}$ or $\mathrm{LOOH}$ (Fig. 8), and that the mechanism of reactions of the Cyt-CL complex in the hydrophobic environment, for all indices, does not differ from the mechanism of peroxidase reactions of the cytochrome c complex with cardiolipin and ordinary peroxidases such as horseradish peroxidase. All these results are consistent with the hypothesis of the existence of Cyt-CL nanospheres in biological membranes (Fig. 9). Studies of the action of the Cyt-CL complex on the membrane structures of mitochondria and cells are needed for further development of this concept.

\section{Conclusion}

This study resulted in establishing the procedure to assemble Cyt-CL complex in non-polar environment for analysis of the complex properties by means of rather simple but powerful methods such as DLS, spectrofluorimetry, and spectrophotometry. First time the self-assembly of the Cyt-CL complex has been studied in non-polar medium (chloroform-methanol medium) mimicking 
microenvironment of a lipid membrane. The results obtained corroborate well with those reported for crystals of the complex analyzed by SAXS. We found that the complex formed in non-polar medium represents three populations of nanoparticles of mean diameters $4.7 \pm 0.7,7.8 \pm 1.0$, and $12.1 \pm 1.4 \mathrm{~nm}$. These particles are likely single molecules of partially unfolded CytC $(4.7 \pm 0.7 \mathrm{~nm})$ and $\mathrm{CytC}$ coated with lipid membrane (other two fractions). The CytC conformation in the Cyt-CL complex changes in non-polar environment as evidenced by: i) increase of CytC hydrodynamic diameter, ii) appearance of Tyr and Trp fluorescence (emission at 315 and $330 \mathrm{~nm}$, respectively), and iii) absence of absorption of the Fe $\cdots>\mathrm{S}$ (Met80) bond at $699 \mathrm{~nm}$. The change in conformation gives cytochrome the quasi-lipoxygenase and lipoperoxidase activity in non-polar solvent. We believe that these results can further support the hypothesis of integration of Cyt-CL complex into the lipid mitochondrial membrane as a core-shell structure. In addition, the developed approach opens new avenues to study the complexation between CytC and CL in non-polar medium that can help to better understand functions of the complex and its substantial role in apoptosis.

Our result section touches on the studies of free protein and the complex in polar and nonpolar environments, and it seems to be difficult to understand how studies under different solvent conditions relate to each other. The answer to this question cannot be done in a general form. (1) Comparing the dimensions of a cytochrome $\mathrm{c}$ molecule in an aqueous solution and in a nanoparticle, we showed that these dimensions differ. This is due to the partial unfolding the protein globule in the Cyt-CL nanosphere. (2) When comparing the data on the properties of the complex Cyt-CL microcrystalls in the suspension and inside a nonpolar solvent, we found that the results were the same, but particles were less uniform in the second case. This is, however, not surprising, since the environment of each nanosphere in both cases was hydrophobic. (3) In a hydrophobic solvent, CytCL particles proved to be catalytically active due to a change in the conformation of cytochrome $c$ in the complex. In the aqueous solution, the Cyt-CL complex is also catalytically active, but this is because cytochrome $c$ is in the hydrophobic environment of phospholipids, and according to our hypothesis the entire Cyt-CL nanoparticle interacting with the oxidation substrate (the liposomes of polyunsaturated phospholipid) penetrates into the lipid bilayer and thus appears in a hydrophobic environment.

\section{Acknowledgment}

This work is supported by Russian Scientific Foundation (grant N 17-74-10248) and by the Alexander von Humboldt Foundation in the framework of the Sofja Kovalevskaja program. 


\section{References}

Antonov, V.F., Puchkov, M.N., Korepanova, E.A., Nemchenko, O.Y., Borodulin, V., 2014. Soft perforation of cardiolipin-containing planar lipid bilayer membrane by cytochrome c and $\mathrm{H}(2) \mathrm{O}(2)$. Eur Biophys J 43, 469-476.

Belikova, N.A., Vladimirov, Y.A., Osipov, A.N., Kapralov, A.A., Tyurin, V.A., Potapovich, M.V., Basova, L.V., Peterson, J., Kurnikov, I.V., Kagan, V.E., 2006. Peroxidase activity and structural transitions of cytochrome c bound to cardiolipin-containing membranes. Biochemistry 45, 4998-5009.

Bergstrom, C.L., Beales, P.A., Yang, Vanderlick, L.Y., , T.K., Groves, J.T., 2013. Cytochrome c causes pore formation in cardiolipin-containing membranes. Proceedings of the National Academy of Sciences 110, 6269-6274.

Brown, L.R., Wuthrich, K., 1977. NMR and ESR studies of the interactions of cytochrome $\mathrm{c}$ with mixed cardiolipinphosphatidylcholine vesicles. Biochim Biophys Acta 468, 389-410.

Burshtein, E.A., Zimina, G.M., Vladimirov, Y.A., 1966. A luminescence method for determining the state of aromatic amino acids (tyrosine and tryptophan) in proteins and enzyme systems. Journal of Applied Spectroscopy 4, 108-110.

Chance, B., 1949. The Properties of the Enzyme-Substrate Compounds of Horse-Radish and Lacto-Peroxidase. Science 109, 204-208.

Chance, B., 1952. The transition from the primary to the secondary peroxidase-peroxide complex. Arch Biochem Biophys 37, 235-237.

Chance, B., Higgins, J., 1952. Peroxidase kinetics in coupled oxidation; an experimental and theoretical study. Arch Biochem Biophys 41, 432-441.

Cheremisina, Z.P., Olenov, V.I., Vladimirov Iu, A., 1972. [Chemoluminescence, linked to the formation of lipid peroxides in biological membranes. 8. Reactions of $\mathrm{Fe} 2+$ and lipid peroxides at the rapid light flash stage]. Biofizika $17,605-610$.

Choi, S., Swanson, J.M., 1995. Interaction of cytochrome c with cardiolipin: an infrared spectroscopic study. Biophys Chem 54, 271-278.

Cormier, M.J., Prichard, P.M., 1968. An investigation of the mechanifm of the luminescent peroxidation of luminol by stopped flow techniques. J Biol Chem 243, 4706-4714.

de Kruijff, B., Cullis, P.R., 1980. Cytochrome c specifically induces non-bilayer structures in cardiolipin-containing model membranes. Biochim Biophys Acta 602, 477-490.

El-Kashef, H., 2000. The necessary requirements imposed on polar dielectric laser dye solvents. Physica B: Condensed Matter 279, 295-301.

Firsov, A.M., Kotova, E.A., Korepanova, E.A., Osipov, A.N., Antonenko, Y.N., 2015. Peroxidative permeabilization of liposomes induced by cytochrome c/cardiolipin complex. Biochimica et biophysica acta 1848, 767-774.

Folch, J., Lees, M., Sloane Stanley, G.H., 1957. A simple method for the isolation and purification of total lipides from animal tissues. J Biol Chem 226, 497-509.

Furtmuller, P.G., Jantschko, W., Zederbauer, M., Jakopitsch, C., Arnhold, J., Obinger, C., 2004. Kinetics of interconversion of redox intermediates of lactoperoxidase, eosinophil peroxidase and myeloperoxidase. Jpn J Infect Dis 57, S30-31.

Hale, G.M., Querry, M.R., 1973. Optical constants of water in the 200-nm to $200-\mu \mathrm{m}$ wavelength region. Applied optics $12,555-563$

Hanske, J., Toffey, J.R., Morenz, A.M., Bonilla, A.J., Schiavoni, K.H., Pletneva, E.V., 2012. Conformational properties of cardiolipin-bound cytochrome c. Proceedings of the National Academy of Sciences 109, 125-130.

Jain, R., Sharma, D., Kumar, R., 2013. Effects of alcohols on the stability and low-frequency local motions that control the slow changes in structural dynamics of ferrocytochrome c. J Biochem 154, 341-354.

Kagan, V.E., Bayir, A., Bayir, H., Stoyanovsky, D., Borisenko, G.G., Tyurina, Y.Y., Wipf, P., Atkinson, J., Greenberger, J.S., Chapkin, R.S., Belikova, N.A., 2009a. Mitochondria-targeted disruptors and inhibitors of cytochrome c/cardiolipin peroxidase complexes: a new strategy in anti-apoptotic drug discovery. Mol Nutr Food Res 53, 104-114.

Kagan, V.E., Bayir, H.A., Belikova, N.A., Kapralov, O., Tyurina, Y.Y., Tyurin, V.A., Jiang, J., Stoyanovsky, D.A., Wipf, P., Kochanek, P.M., Greenberger, J.S., Pitt, B., Shvedova, A.A., Borisenko, G., 2009b. Cytochrome c/cardiolipin relations in mitochondria: a kiss of death. Free Radic Biol Med 46, 1439-1453.

Kagan, V.E., Borisenko, G.G., Tyurina, Y.Y., Tyurin, V.A., Jiang, J., Potapovich, A.I., Kini, V., Amoscato, A.A., Fujii, Y., 2004. Oxidative lipidomics of apoptosis: redox catalytic interactions of cytochrome $\mathrm{c}$ with cardiolipin and phosphatidylserine. Free Radic Biol Med. 37, 1963-1985.

Kagan, V.E., Tyurin, V.A., Jiang, J., Tyurina, Y.Y., Ritov, V.B., Amoscato, A.A., Osipov, A.N., Belikova, N.A., Kapralov, A.A., Kini, V.V., Vlasova, I.I., Zhao, Q., Zou, M., Di, P., Svistunenko, D.A., Kurnikov, I.V., Borisenko, G.G., 2005. Cytochrome $c$ acts as a cardiolipin oxygenase required for release of proapoptotic factors. Nature Chem Biol 1 , 223-232.

Kapralov, A.A., Kurnikov, I.V., Vlasova, I.I., Belikova, N.A., Tyurin, V.A., Basova, L.V., Zhao, Q., Tyurina, Y.Y., Jiang, J., Bayir, H., Vladimirov, Y.A., Kagan, V.E., 2007. The hierarchy of structural transitions induced in cytochrome 
$c$ by anionic phospholipids determines its peroxidase activation and selective peroxidation during apoptosis in cells. Biochemistry 46, 14232-14244.

Kapralov, A.A., Yanamala, N., Tyurina, Y.Y., Castro, L., Samhan-Arias, A., Vladimirov, Y.A., Maeda, A., Weitz, A.A., Peterson, J., Mylnikov, D., Demicheli, V., Tortora, V., Klein-Seetharaman, J., Radi, R., Kagan, V.E., 2011. Topography of tyrosine residues and their involvement in peroxidation of polyunsaturated cardiolipin in cytochrome c/cardiolipin peroxidase complexes. Biochimica et Biophysica Acta 1808, 2147-2155.

Kedenburg, S., Vieweg, M., Gissibl, T., Giessen, H., 2012. Linear refractive index and absorption measurements of nonlinear optical liquids in the visible and near-infrared spectral region. Optical Materials Express 2, 1588-1611.

Kestin, J., Sokolov, M., Wakeham, W.A., 1978. Viscosity of liquid water in the range- 8 C to 150 C. Journal of Physical and Chemical Reference Data 7, 941-948.

Kobayashi, H., Nagao, S., Hirota, S., 2016. Characterization of the Cytochrome c Membrane-Binding Site Using Cardiolipin-Containing Bicelles with NMR. Angew Chem Int Ed Engl 55, 14019-14022.

Konev, S.V., 1967. Fluorescence and phosphorescence of proteins and nucleic acids. Plenum Press, New York,.

Lee, J.C., Engman, K.C., Tezcan, F.A., Gray, H.B., Winkler, J.R., 2002. Structural features of cytochrome c' folding intermediates revealed by fluorescence energy-transfer kinetics. Proceedings of the National Academy of Sciences 99, 14778-14782.

Mandal, A., Hoop, C.L., DeLucia, M., Kodali, R., Kagan, V.E., Ahn, J., van der Wel, P.C., 2015. Structural Changes and Proapoptotic Peroxidase Activity of Cardiolipin-Bound Mitochondrial Cytochrome c. Biophys J 109, 1873-1884.

Maniti, O., Cheniour, M., Lecompte, M.-F., Marcillat, O., Buchet, R., Vial, C., Granjon, T., 2011. Acyl chain composition determines cardiolipin clustering induced by mitochondrial creatine kinase binding to monolayers. Biochimica et Biophysica Acta (BBA)-Biomembranes 1808, 1129-1139.

Marchenkova, M.A., Dyakova, Y.A., Tereschenko, E.Y., Kovalchuk, M.V., Vladimirov, Y.A., 2015. Cytochrome c Complexes with Cardiolipin Monolayer Formed under Different Surface Pressure. Langmuir 31, 12426-12436.

McClelland, L.J., Steele, H.B., Whitby, F.G., Mou, T.C., Holley, D., Ross, J.B., Sprang, S.R., Bowler, B.E., 2016. Cytochrome c Can Form a Well-Defined Binding Pocket for Hydrocarbons. J Am Chem Soc 138, 16770-16778.

Narendra, K., Narayanamurthy, P., Srinivasu, C., 2011. Refractive indices of binary liquid mixture at different temperatures. Asian J. Applied Sci 4, 535-541.

O'Brien, E.S., Nucci, N.V., Fuglestad, B., Tommos, C., Wand, A.J., 2015. Defining the Apoptotic Trigger: THE INTERACTION OF CYTOCHROME c AND CARDIOLIPIN. J Biol Chem 290, 30879-30887.

Osipov, A.N., Stepanov, G.O., Vladimirov, Y.A., Kozlov, A.V., Kagan, V.E., 2006. Regulation of cytochrome C peroxidase activity by nitric oxide and laser irradiation. Biochemistry (Mosc) 71, 1128-1132.

Pandiscia, L.A., Schweitzer-Stenner, R., 2015. Coexistence of native-like and non-native partially unfolded ferricytochrome $\mathrm{c}$ on the surface of cardiolipin-containing liposomes. J Phys Chem B 119, 1334-1349.

Patriarca, A., Eliseo, T., Sinibaldi, F., Piro, M.C., Melis, R., Paci, M., Cicero, D.O., Polticelli, F., Santucci, R., Fiorucci, L., 2009. ATP acts as a regulatory effector in modulating structural transitions of cytochrome c: implications for apoptotic activity. Biochemistry 48, 3279-3287.

Proskurnina, E.V., Alekseev, A.V., Demin, E.M., Izmailov, D.Y., Vladimirov, Y.A., 2013. Cyt-CL complex: Peroxidase activity and role in lipid peroxidation. FEBS JOURNAL 280, 264-264.

Rytomaa, M., Kinnunen, P.K., 1995. Reversibility of the binding of cytochrome $\mathrm{c}$ to liposomes. Implications for lipidprotein interactions. J Biol Chem 270, 3197-3202.

Shimada, O., Yasuda, H., 1977. Lipid peroxidation and its inhibition by tinoridine. I. Lipid peroxidation-induced disintegration of microsomal membrane and cytochrome P-450 in rat liver. Biochim Biophys Acta 489, 163-172.

Shtykova, E., Volkov, V., Remenschikov, V., Vladimirov, Y., 2013. Probing a Complex of Cytochrome c and Cardiolipin by SAXS in Solution and in Precipitate, 11th International Conference "Biology and Synchrotron Radiation". EMBL. Poster Abstracts, Hamburg, Germany, p. 153.

Sinibaldi, F., Fiorucci, L., Patriarca, A., Lauceri, R., Ferri, T., Coletta, M., Santucci, R., 2008. Insights into cytochrome c-cardiolipin interaction. Role played by ionic strength. Biochemistry 47, 6928-6935.

Skulachev, V.P., 1998. Cytochrome $\mathrm{c}$ in the apoptotic and antioxidant cascades. FEBS Lett 423, 275-280.

Snider, E.J., Muenzner, J., Toffey, J.R., Hong, Y., Pletneva, E.V., 2013. Multifaceted effects of ATP on cardiolipinbound cytochrome c. Biochemistry 52, 993-995.

Spooner, P.J., Watts, A., 1991a. Reversible unfolding of cytochrome c upon interaction with cardiolipin bilayers. 1. Evidence from deuterium NMR measurements. Biochemistry 30, 3871-3879.

Spooner, P.J., Watts, A., 1991b. Reversible unfolding of cytochrome c upon interaction with cardiolipin bilayers. 2. Evidence from phosphorus-31 NMR measurements. Biochemistry 30, 3880-3885.

Stepanov, G., Gnedenko, O., Mol'nar, A., Ivanov, A., Vladimirov, Y., Osipov, A., 2009. Evaluation of cytochrome c affinity to anionic phospholipids by means of surface plasmon resonance. FEBS Lett 583, 97-100. 
Stetefeld, J., McKenna, S.A., Patel, T.R., 2016. Dynamic light scattering: a practical guide and applications in biomedical sciences. Biophys Rev 8, 409-427.

Tyurina, Y.Y., Poloyac, S.M., Tyurin, V.A., Kapralov, A.A., Jiang, J., Anthonymuthu, T.S., Kapralova, V.I., Vikulina, A.S., Jung, M.-Y., Epperly, M.W., Mohammadyani, D., Klein-Seetharaman, J., Jackson, T.C., Kochanek, P.M., Pitt, B.R., Greenberger, J.S., Vladimirov, Y.A., Bayir, H., Kagan, V.E., 2014. A mitochondrial pathway for biosynthesis of lipid mediators. Nature chemistry

6, 542-552.

Vikulina, A.S., Alekseev, A.V., Proskurnina, E.V., Vladimirov, Y.A., 2015. Cytochrome c-Cardiolipin Complex in a Nonpolar Environment. Biochemistry (Moscow) 80, 1298-1302.

Vladimirov, I.A., Suslova, T.B., Olenev, V.I., 1969. [Chemiluminescence associated with the formation of lipid peroxides in biological membranes. II. The role of $\mathrm{Fe} 2+$ in the development of chain oxidation of lipids and of ultraweak luminescence]. Biofizika 14, 836-845.

Vladimirov, I.A., Zimina, G.M., 1965. [The luminescence of some proteins and of tryptophan under monochromatic excitation in solutions of different pH]. Biokhimiia (Moscow, Russia) 30, 1105-1114.

Vladimirov, I.U.A., 1969. Photochemistry and luminescence of proteins. Israel Program for Scientific Translations available from the U.S. Dept. of Commerce, Clearinghouse for Federal Scientific and Technical Information, Springfield, Va., Jerusalem,.

Vladimirov, Y.A., 1996. Intrinsic (low-level) chemiluminescence, In: Punchard, N.A.a.K., F.J. (Ed.), Free radicals. A practical approach. Oxford University Press, Oxford, New York, Tokyo, pp. 65-82.

Vladimirov, Y.A., Alekseev, A.V., Volkov, V.V., Remenschikov, V.E., Proskurnina, E.V., 2013a. The cytochrome c forms a complex with cardiolipin in a form of hydrophobic nanospheres. The FEBS journal 280, 263-264.

Vladimirov, Y.A., Archakov, A.I., 1972. Lipid peroxidation in biological membranes (Rus). Nauka, Moscow.

Vladimirov, Y.A., Demin, E.M., Proskurnina, E.V., Osipov, A.N., 2009a. Lipoperoxide radical production during oxidation of cardiolipin in the complex with cytochrome $c$. Biochemistry (Moscow) Supplement Series A: Membrane and Cell Biology 3, 479-489.

Vladimirov, Y.A., Nol', Y.T., Volkov, V.V., 2011. Protein-lipid nanoparticles that determine whether cells will live or die. Crystallography Reports 56, 553-559.

Vladimirov, Y.A., Olenev, V.I., Suslova, T.B., Cheremisina, Z.P., 1980. Lipid peroxidation in mitochondrial membrane. Advances in Lipid Research 17, 173-249.

Vladimirov, Y.A., Proskurnina, E.V., 2009. Free Radicals and Cell Chemiluminescence. Biochemistry (Moscow) 74, 1545-1566.

Vladimirov, Y.A., Proskurnina, E.V., Alekseev, a.V., 2013b. Molecular Mechanisms of Apoptosis. Structure of Cytochrome c-Cardiolipin Complex. Biochemistry. Biokhimiia 78, 1086-1097.

Vladimirov, Y.A., Proskurnina, E.V., Demin, E.M., Matveeva, N.S., Lubitskiy, O.B., Novikov, A.A., Izmailov, D.Y., Osipov, A.N., Tikhonov, V.P., Kagan, V.E., 2009b. Dihydroquercetin (taxifolin) and other flavonoids as inhibitors of free radical formation at key stages of apoptosis. Biochemistry (Mosc) 74, 301-307.

Vladimirov, Y.A., Proskurnina, E.V., Izmailov, D.Y., Novikov, A.A., Brusnichkin, A.V., Osipov, A.N., Kagan, V.E., 2006a. Cardiolipin activates cytochrome c peroxidase activity since It facilitates $\mathrm{H}_{2} \mathrm{O}_{2}$ access to heme Iron. Biochemistry (Moscow) 71, 1225-1233.

Vladimirov, Y.A., Proskurnina, E.V., Izmailov, D.Y., Novikov, A.A., Brusnichkin, A.V., Osipov, A.N., Kagan, V.E., 2006b. Cardiolipin activates cytochrome c peroxidase activity since it facilitates $\mathrm{H}(2) \mathrm{O}(2)$ access to heme. Biochemistry (Mosc) 71, 998-1005.

Vladimirov, Y.A., Proskurnina, E.V., Izmailov, D.Y., Novikov, A.A., Brusnichkin, A.V., Osipov, A.N., Kagan, V.E., 2006c. Mechanism of activation of cytochrome C peroxidase activity by cardiolipin. Biochemistry (Mosc) 71, 989-997. Vladimirov, Y.A., Sarisozen, C., Vladimirov, G.K., Filipczak, N., Polimova, A.M., Torchilin, V.P., 2017. The Cytotoxic Action of Cytochrome C/Cardiolipin Nanocomplex (Cyt-CL) on Cancer Cells in Culture. Pharm Res 34, 1264-1275.

Vladimirov, Y.A., Sharov, V.S., Driomina, E.S., Reznitchenko, A.V., Gashev, S.B., 1995. Coumarin derivatives enhance the chemiluminescence accompanying lipid peroxidation. Free Radic Biol Med 18, 739-745.

Wei, W., Danielson, N.D., 2011. Fluorescence and circular dichroism spectroscopy of cytochrome c in alkylammonium formate ionic liquids. Biomacromolecules 12, 290-297.

Yilmaz, H., 2002. Excess properties of alcohol-water systems at 298.15 K. Turkish Journal of Physics 26, 243-246. 
Graphical abstract
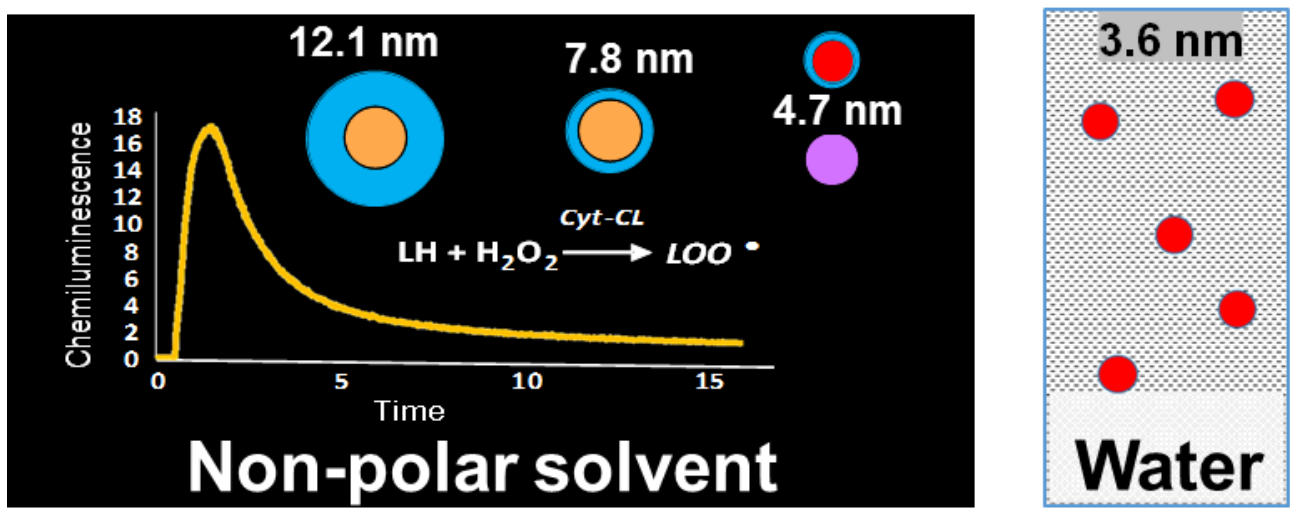